\title{
Optimal Control Techniques on a Mathematical Model for the Dynamics of Tungiasis in a Community
}

\author{
Jairos Kahuru, ${ }^{1}$ Livingstone S. Luboobi, ${ }^{1,2}$ and Yaw Nkansah-Gyekye ${ }^{1}$ \\ ${ }^{1}$ School of Computational and Communication Science and Engineering, \\ Nelson Mandela African Institution of Science and Technology, P.O. Box 447, Arusha, Tanzania \\ ${ }^{2}$ Department of Mathematics, Makerere University, P.O. Box 7062, Kampala, Uganda \\ Correspondence should be addressed to Jairos Kahuru; shinzehk@nm-aist.ac.tz
}

Received 20 March 2017; Accepted 2 July 2017; Published 14 August 2017

Academic Editor: Nawab Hussain

Copyright (C) 2017 Jairos Kahuru et al. This is an open access article distributed under the Creative Commons Attribution License, which permits unrestricted use, distribution, and reproduction in any medium, provided the original work is properly cited.

\begin{abstract}
Tungiasis is a permanent penetration of female sand flea "Tunga penetrans" into the epidermis of its host. It affects human beings and domestic and sylvatic animals. In this paper, we apply optimal control techniques to a Tungiasis controlled mathematical model to determine the optimal control strategy in order to minimize the number of infested humans, infested animals, and sand flea populations. In an attempt to reduce Tungiasis infestation in human population, the control strategies based on personal protection, personal treatment, educational campaign, environmental sanitation, and insecticidal treatments on the affected parts as well as on animal fur are considered. We prove the existence of optimal control problem, determine the necessary conditions for optimality, and then perform numerical simulations. The numerical results showed that the control strategy comprises all five control measures and that which involves the three control measures of insecticide control, insecticidal dusting on animal furs, and environmental hygiene has the significant impact on Tungiasis transmission. Therefore, fighting against Tungiasis infestation in endemic settings, multidimensional control process should be employed in order to achieve the maximum benefits.
\end{abstract}

\section{Introduction}

Tungiasis is a skin disease caused by the sand flea "Tunga penetrans"; the disease is endemic in some poor resource communities where various domestic and sylvatic animals act as reservoirs for this zoonosis [1]. The flea infestation is associated with poverty and occurs in many resource-poor communities in the Caribbean, South America, and subSaharan Africa [2]. Transmission of Tungiasis is strictly by infestation of humans and animal reservoirs by "Tunga penetrans" when they are in contact with sandy soil in which female fleas are present or when in contact with infested animal reservoirs as it is known that the animal reservoirs harbor the fleas [3]. Tungiasis results in significant morbidity, manifesting itself in a number of symptoms such as severe local inflammation, autoamputation of digits, deformation and loss of nails, formation of fissures and ulcers, gangrene, and walking difficulties [4]. Moreover it may result into secondary infection caused by transmission of blood-borne pathogens such as hepatitis $\mathrm{B}$ and $\mathrm{C}$ virus and possibly also
HIV/AIDS when a single nonsterile instrument is used to remove the jiggers from different affected individuals [5].

Mathematical models have played a major role in increasing understanding of the underlying mechanisms which influence the spread of the diseases and provide guidelines as to how the spread can be controlled [6,7]. Optimal control theory is a powerful mathematical tool which makes the decision involving complex dynamical systems. It is a standard method for solving dynamic optimization problems, when those problems are expressed in continuous time [8]. Optimal control theory was developed by the Russian mathematician Lev S. Pontryagin (1908-1988) and his coworkers with the formulation and proof of the Pontryagin Maximum Principle (Pontryagin et al., 1962). Optimal control is the process of determining control and state trajectories for a dynamic system over a period of time to minimize a performance index [9]. Optimal control problem is represented by a set of differential equations describing the paths of the control variables that minimize the cost functional and has been used successfully to make decisions involving biological or 
medical models [10]. The formulation of an optimal control problem requires a mathematical model of the system to be controlled, a specification of the performance index (cost function), and a specification of all boundary conditions on states and constraints to be satisfied by states and controls [11]. Pontryagin's maximum (or minimum) principle of optimal control gives the fundamental necessary conditions for a controlled trajectory to be optimal [12]. The principle technique is to transform the constrained dynamic optimization problem into an unconstrained problem, by allowing each of the adjoint variables to correspond to each of the state variables accordingly and combining the results with the objective functional [13]. The resulting function is known as the Hamiltonian, which is used to solve a set of necessary conditions that an optimal control and corresponding state variables must satisfy. The necessary conditions are the optimality solutions and adjoint equations which form the optimality system. The optimality system consists of the state system and adjoint system with initial and transversal conditions together with characterization of optimal control.

To the best of our knowledge Tungiasis dynamical model with application of optimal control technique has not been done. Therefore we are going to refer to other infectious diseases with similar characteristics where the optimal control theory has been applied. Bonyah et al. [20] applied optimal control theory to a Buruli ulcer model that takes into account human, water bug, and fish populations as well as Microbacterium ulcerans in the environment. The control measures were applied on mass treatment, insecticide, and mass education to minimize the number of infected hosts, vectors, and infected fishes. The optimality system was determined and computed numerically for several scenarios. The results showed that the combination of all the control measures, mass treatment, insecticide, and mass education, is capable of helping reduce the number of infected humans, water bugs, small fishes, and Mycobacterium ulcerans in the environment. Isere et al. [21] developed the optimal control model that includes two time dependent control functions with one minimizing the contact between the susceptible and the bacteria and the others, the population of bacteria in water. The results from the numerical solutions showed that increasing the susceptible pool and the infected populations above some threshold values were responsible for reducing cholera epidemic and the difference between the growth rate and the loss rate of the bacteria played a huge role in the outbreak of the disease. Devipriya and Kalaivani [22] conducted the study on "Optimal Control of Multiple Transmission of WaterBorne Disease." A controlled SIWR model was considered. The control measures represented an immune boosting and pathogen suppressing drugs. Their objective function was based on a combination of minimizing the number of infected individuals and the cost of the drugs dose. The numerical results have shown that both the vaccines resulted in minimizing the number of infected individuals and at the same time in a reduction of the budget related to the disease.

In this paper, the Tungiasis dynamical model with control measures is presented and a detailed qualitative optimal control model that minimizes the number of infested individuals (humans and animals) and sand fleas with minimal cost of implementing the control measures is developed. We establish the proof for existence of the optimal control and analyze the optimal control problem in order to determine the necessary conditions for optimality using the Pontryagin's maximum principle (Pontryagins et al., 1962). We then determine numerically the optimality system for several scenarios. Our paper is arranged as follows. In Section 2, we formulate an optimal control model. In Section 3, we analyze the optimal control model by determining the conditions for existence of optimal control and the necessary conditions for optimality. In Section 4, we carry out numerical simulations and discussion of the results and Section 5 is the conclusion.

\section{Formulation of Optimal Control Problem}

We formulate an optimal control model for Tungiasis disease in order to derive five optimal control measures with minimal implementation cost to eradicate the disease after a defined period of time. We employ the control efforts $w_{i}(t)$ in human, animal reservoirs, and adult flea populations and $\left(1-w_{i}(t)\right)$ is the failure rate for the control efforts $w_{i}(t)$ for $i=$ $1, \ldots, 5$. We let $w_{1}(t)$ be the effort of controlling the flea infested soil environment with insecticides spraying, $w_{2}(t)$ be the efforts of controlling the flea infested animal reservoir through dusting them with ant-flea compounds, $w_{3}(t)$ be the efforts of controlling the transmission from flea infested environment to susceptible animals (this can be achieved by environmental hygiene and cementing the floors), $w_{4}(t)$ be the efforts of controlling the transmission from flea infested animals to susceptible humans (this can be achieved by educating people not to live with animals in the same quarters or sharing common resting places), and $w_{5}(t)$ be the efforts of controlling transmission from flea infested environment to susceptible humans (this can be achieved by environmental hygiene, cementing the floors, covering of feet with solid shoes, and application of plant based repellent (Zanzarin) within the time interval of $[0, T])$. Therefore we assume that the mortality rate of jigger fleas in the soil environment is increased by the factor $\left(\mu_{F}+w_{1}(t)\right)$, on-host spraying of infested animals will reduce the shedding rate $\varepsilon_{A}$ of adult jigger fleas into the environment by a fraction $\left(1-w_{2}(t)\right)$, and the animal to animal effective contact rate $\rho_{A}$ is reduced at the same fraction $\left(1-w_{2}(t)\right)$ because spraying insecticides on animal fur will reduce the transmission of infestation within animal population. The transmission rate from the soil environment to animal hosts is reduced by the factor $\left(1-w_{3}(t)\right)$, the factor $\left(1-w_{4}(t)\right)$ reduces the transmission from severely infested animal reservoirs to susceptible humans, and the factor $\left(1-w_{5}(t)\right)$ reduces the transmission from flea infested soil environment to susceptible humans. Here, we consider the model developed by Kahuru et al. [19] whereby we add a distinct epidemiological compartment $T_{H}$ which represents human beings under treatment and incorporate the five control measures $w_{1}, w_{2}, w_{3}, w_{4}$, and $w_{5}$ as defined above. In the submodel of human population, the total human population $N_{H}$ is subdivided into susceptible population $S_{H}$ mildly infested population $I_{H l}$, the severely infested population $I_{H h}$, and the human treatment class denoted by 
$T_{H}$; therefore we have $N_{H}=S_{H}+I_{H l}+I_{H h}+T_{H}$. We assume that the humans are recruited into $S_{H}$ through birth by the adults at a rate $b_{H}$. Individuals in class $S_{H}$ acquire infestation from the severely infested animal reservoirs $I_{A h}$ and move to class $I_{H l}$ at a rate $\left(1-w_{4}(t)\right) \rho_{A H} I_{A h} / N_{H}$ and may also acquire infestation from the flea infested soil environment and move to class $I_{H h}$ at a rate $\left(1-w_{5}(t)\right) \alpha_{E H} \beta_{E H} r_{F} F_{E} /\left(k+F_{E}\right)$. $I_{H I}$ may as well acquire infestation from the flea infested soil environment and progresses to class $I_{H h}$ at a rate (1 $\left.w_{5}(t)\right) \alpha_{E H} \beta_{E H} r_{F} F_{E} /\left(k+F_{E}\right)$. Classes $I_{H h}$ and $I_{H l}$ seek treatment at the respective rates $p_{1}$ and $p_{2}$ and join $T_{H}$ class, and eventually the treated individuals revert back to join $S_{H}$ at a progression rate $\omega$. Individuals in compartments $S_{H}, I_{H l}$, and $T_{H}$ suffer a natural mortality rate $\mu_{H}$ and for the compartment $I_{H h}$ they suffer a natural mortality at a rate $\mu_{H}$ and the disease induced mortality at a rate $\sigma_{H}$. In the submodel of animal reservoir population, the total animal reservoir population $N_{A}$ is subdivided into susceptible population $S_{A}$ mildly infested population $I_{A l}$ and the severely infested population $I_{A h}$; therefore we have $N_{A}=S_{A}+I_{A l}+I_{A h}$. We assume that the animals are recruited into $S_{A}$ through birth by the adults at a rate $b_{A}$. Individuals in class $S_{A}$ acquire infestation from the severely infested animal reservoirs $I_{A h}$ and move to $I_{A l}$ at a rate $\left(1-w_{2}(t)\right) \rho_{A} I_{A h} / N_{A}$ and also may acquire infestation from the flea infested environment and move to class $I_{A h}$ at a rate $\left(1-w_{3}(t)\right) \alpha_{E A} \beta_{E A} r_{F} F_{E} /\left(k+F_{E}\right)$. $I_{A l}$ may as well acquire infestation from the flea infested soil environment and progresses to class $I_{A h}$ at a rate (1$\left.w_{3}(t)\right) \alpha_{E A} \beta_{E A} r_{F} F_{E} /\left(k+F_{E}\right)$. Individuals in compartments $S_{A}$ and $I_{A l}$ suffer a natural mortality rate $\mu_{A}$ and for the compartment $I_{A h}$ they suffer a natural mortality at a rate $\mu_{A}$ and a disease induced mortality at a rate $\sigma_{A}$. The submodel of environmental component consists of two compartments, a compartment of larvae denoted by $L_{E}$ and a compartment of adult sand fleas denoted by $F_{E}$. The larvae population are recruited into $L_{E}$ through shedding of jigger eggs by $I_{H h}$ and $I_{A h}$ at a constant rate $\delta_{e}$; therefore we have the total contribution of $\delta_{e} I_{H h}$ and $\delta_{e} I_{A h}$ from infested humans and animal reservoir populations, respectively. The larvae in compartment $L_{E}$ mature into adult jigger fleas at a maturation rate $\gamma_{L}$ and undergo a natural death at a rate $\mu_{L}$. The adult jigger flea population are recruited into $F_{E}$ through maturation by larvae at a rate $\gamma_{L}$ and from infested animal reservoirs who contributes the fleas into the soil environment at a rate $\left(1-w_{2}(t)\right) \varepsilon_{A}$. The adult fleas leave the compartment $F_{E}$ when they attack the hosts at a rate $r_{F} F_{E} /\left(k+F_{E}\right)$ and when they undergo a natural death at a rate $\mu_{F}$ and the additional death due to insecticides control at a rate $w_{1}(t)$, therefore we have the flea total death rate of $\left(\mu_{F}+w_{1}(t)\right) F_{E}$.

The variables and parameters that describe the flow rates between compartments are given, respectively, in Notations. The possible interactions between humans, animal reservoirs, and flea infested environment with control measures are presented by the model flow diagram in Figure 1 and the differential equations describing the model are given in system (1).

2.1. Model Flow Chart with Control Measures. The dynamical model with submodels of humans, animal reservoirs, and flea infested environment that incorporates time dependent control measures is presented hereunder.

2.2. Equations of the Model with Control Measures. From compartmental flow chart in Figure 1 the nonlinear differential equations representing the controlled system of Tungiasis dynamical model are given by

Dynamics in human population

$$
\begin{aligned}
& \frac{d S_{H}(t)}{d t} \\
& =b_{H} N_{H}+\omega T_{H}-\left(1-w_{5}(t)\right) \psi_{E H} S_{H} \\
& \quad-\left(1-w_{4}(t)\right) \psi_{A H} S_{H}-\mu_{H} S_{H} \\
& \frac{d I_{H l}(t)}{d t} \\
& =\left(1-w_{4}(t)\right) \psi_{A H} S_{H}-\left(1-w_{5}(t)\right) \psi_{E H} I_{H l} \\
& \quad-\left(p_{1}+\mu_{H}\right) I_{H l} \\
& \frac{d I_{H h}(t)}{d t} \\
& =\left(1-w_{5}(t)\right) \psi_{E H} S_{H}+\left(1-w_{5}(t)\right) \psi_{E H} I_{H l} \\
& \quad-\left(p_{2}+\mu_{H}+\sigma_{H}\right) I_{H h} \\
& \frac{d T_{H}(t)}{d t}=p_{1} I_{H l}+p_{2} I_{H h}-\left(\omega+\mu_{H}\right) T_{H}
\end{aligned}
$$

Dynamics in animal reservoir population

$$
\begin{aligned}
& \frac{d S_{A}(t)}{d t} \\
& =b_{A} N_{A}-\left(1-w_{2}(t)\right) \psi_{A A} S_{A}-\left(1-w_{3}(t)\right) \psi_{E A} S_{A} \\
& \quad-\mu_{A} S_{A} \\
& \frac{d I_{A l}(t)}{d t} \\
& =\left(1-w_{2}(t)\right) \psi_{A A} S_{A}-\left(1-w_{3}(t)\right) \psi_{E A} I_{A l}-\mu_{A} I_{A l} \\
& \frac{d I_{A h}(t)}{d t} \\
& =\left(1-w_{3}(t)\right) \psi_{E A} S_{A}+\left(1-w_{3}(t)\right) \psi_{E A} I_{A l} \\
& \quad-\left(\mu_{A}+\sigma_{A}\right) I_{A h}
\end{aligned}
$$

Dynamics in jigger flea population

$$
\begin{aligned}
& \frac{d L_{E}(t)}{d t}=\delta_{e}\left(1-\frac{L_{E}}{K}\right)\left(I_{H h}+I_{A h}\right)-\left(\gamma_{L}+\mu_{L}\right) L_{E} \\
& \frac{d F_{E}(t)}{d t} \\
& =\gamma_{L} L_{E}+\left(1-w_{2}(t)\right) \varepsilon_{A} I_{A h}-\left(\mu_{F}+w_{1}(t)\right) F_{E} \\
& \quad-r_{F} \frac{F_{E}}{k+F_{E}}
\end{aligned}
$$




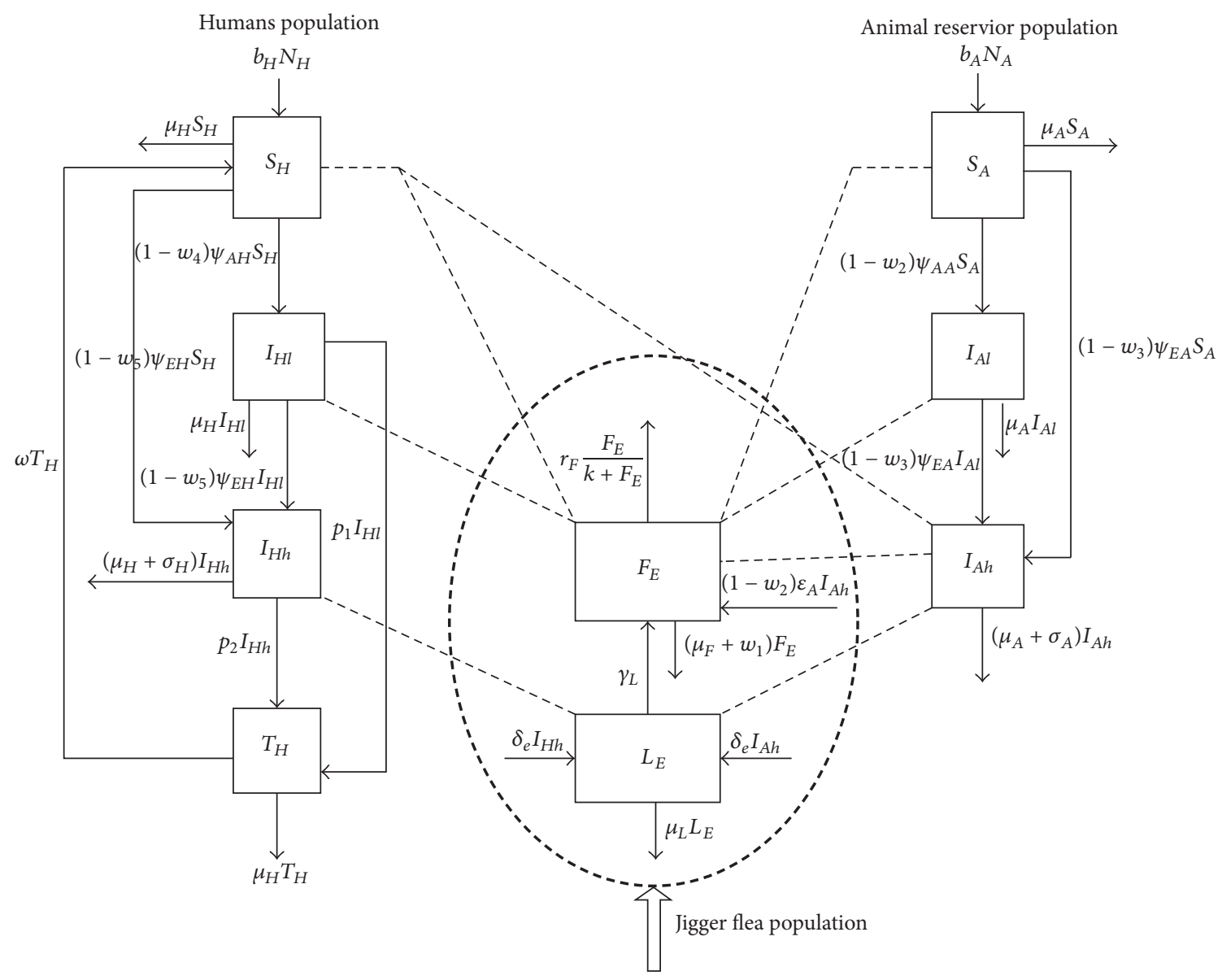

FIGURE 1: The flow chart showing the dynamics of Tungiasis with control measures incorporated.

with initial condition

$$
\begin{gathered}
S_{H}(0)>0, \\
I_{H l}(0) \geq 0, \\
I_{H h}(0) \geq 0, \\
T_{H}(0) \geq 0, \\
S_{A}(0)>0, \\
I_{A l}(0) \geq 0, \\
I_{A h}(0) \geq 0, \\
L_{E}(0) \geq 0, \\
F_{E}(0)>0, \\
w_{1}(t) \geq 0, \\
w_{2}(t) \geq 0, \\
w_{3}(t) \geq 0, \\
w_{4}(t) \geq 0, \\
w_{5}(t) \geq 0,
\end{gathered}
$$

where

$$
\begin{aligned}
\psi_{A A} & =\rho_{A} \frac{I_{A h}}{N_{A}}, \\
\psi_{E A} & =\alpha_{E A} \beta_{E A} r_{F} \frac{F_{E}}{k+F_{E}}, \\
\psi_{E H} & =\alpha_{E H} \beta_{E H} r_{F} \frac{F_{E}}{k+F_{E}}, \\
\psi_{A H} & =\rho_{A H} \frac{I_{A h}}{N_{H}} \\
0 & \leq w_{1}(t), w_{2}(t), w_{3}(t), w_{4}(t), w_{5}(t) \leq 1 .
\end{aligned}
$$

2.3. Optimal Control Problem for Tungiasis Epidemic. In this section, we present the optimal control problem considering the performance index and the controlled model equations with initial state conditions whereby the goal is to find the optimal levels of the control measures needed to minimize the number of infested humans, animal reservoirs, and fleas as well as the cost of implementing the control strategies $\left(w_{i}\right.$ for $i=1, \ldots, 5)$. The objective functional $J(w)$ to be minimized is given by 


$$
J(w)=\min \int_{0}^{\mathrm{T}}\left\{R_{1} I_{H h}+R_{2} I_{H l}+R_{3} I_{A h}+R_{4} I_{A l}+R_{5} F_{E}+\frac{1}{2} \sum_{i=1}^{5} Z_{i} w_{i}^{2}\right\} d t
$$

The terms $I_{H h}, I_{H l}, I_{A h}, I_{A l}, F_{E}$ in the objective functional $J(w)$ are the number of infested populations that need to be minimized. The terms $Z_{1} w_{1}^{2}$ represents the cost of offhost insecticides spraying, $Z_{2} w_{2}^{2}$ represents the cost of onhost spraying of infested domestic animals, $Z_{3} w_{3}^{2}$ represent the cost of implementing environmental hygiene, $Z_{4} w_{4}^{2}$ represents the cost of education campaign, and $Z_{5} w_{5}^{2}$ represents the cost of personal protection. $R_{1}, R_{2}, R_{3}, R_{4}, R_{5}, Z_{1}, Z_{2}, Z_{3}$, $Z_{4}$, and $Z_{5}$ are positive balancing coefficients (weights) which regularize the optimal control. Quadratic expressions of the controls are included to indicate nonlinear costs potentially arising at high intervention levels [23].

The optimal control problem is formulated to obtain the minimum number of infested populations $I_{H h}, I_{H l}, I_{A h}, I_{A l}$, $F_{E}$ under minimum cost. Therefore the objective function in (4) is minimized subject to the model equations in (1). We seek the optimal controls $w_{1}^{*}, w_{2}^{*}, w_{3}^{*}, w_{4}^{*}, w_{5}^{*}$ such that

$$
\begin{aligned}
J & \left(w_{1}^{*}, w_{2}^{*}, w_{3}^{*}, w_{4}^{*}, w_{5}^{*}\right) \\
\quad & =\min J\left\{\left(w_{1}, w_{2}, w_{3}, w_{4}, w_{5}\right) \mid w_{i} \in \Pi \text { for } i\right. \\
& =1, \ldots, 5\}
\end{aligned}
$$

subject to the dynamical system in (1)

and the control set given by

$\Pi=\left\{\left(w_{1}, w_{2}, w_{3}, w_{4}, w_{5}\right)\right.$ such that; $0 \leq w_{i} \leq 1$, for $i$

$$
=1, \ldots, 5 ; \forall t \in[0, T]\} \text {. }
$$

\section{Model Analysis}

The basic framework of an optimal control is to prove the existence of the optimal control and then characterize the optimal control through optimality system [24]. Given the optimal control problem in (5) we prove the existence of optimal control problem using the approach by Fleming and Rishel [25] and by Lukes [26] and then characterizing it for optimality.

3.1. The Existence of Optimal Control Problem. To prove the existence of optimal control the following conditions should be satisfied:

(i) The set of controls and corresponding state variables are nonempty.

(ii) The control set is convex and closed.

(iii) The right-hand side of the state system is bounded by a linear function in the state and control variables.

(iv) The integrand of the objective functional is convex.

(v) The integrand of the objective functional is bounded below by $\tau_{1}\left(\sum_{i=1}^{5}\left|w_{i}\right|^{2}\right)^{\omega / 2}-\tau_{2}$, where $\tau_{1}, \tau_{2}>0$ and $\omega>1$.

Given that, $S_{H}, I_{H l}, I_{H h}, T_{H}, S_{A}, I_{A l}, I_{A h}, L_{E}$, and $F_{E}$ are the state variables of the controlled model system (1) and $w_{1}$, $w_{2}, w_{3}, w_{4}, w_{5}$ are the control variables. We state and prove Theorem 1 as follows.

Theorem 1. There exists an optimal control variables set $w^{*}=$ $\left(w_{1}^{*}, w_{2}^{*}, w_{3}^{*}, w_{4}^{*}, w_{5}^{*}\right) \in \Pi$ such that

$$
J\left(w_{1}^{*}, w_{2}^{*}, w_{3}^{*}, w_{4}^{*}, w_{5}^{*}\right)=\min \quad J\left\{\left(w_{1}, w_{2}, w_{3}, w_{4}, w_{5}\right) \mid w_{i} \in \Pi \text { for } i=1, \ldots, 5\right\}
$$

Proof. Using the results in Fleming and Rishel [25] and by Lukes [26], the control and the state variables are nonnegative values. In this minimization problem, the necessary convexity of the objective functional in $w_{1}, w_{2}, w_{3}, w_{4}, w_{5}$ is satisfied. The set of admissible Lebesgue measurable control variables $\left(w_{1}, w_{2}, w_{3}, w_{4}, w_{5}\right) \in \Pi$ is also convex and closed by the definition. The optimal system is bounded which determines the compactness needed for the existence of optimal control. In order to verify this argument we use the approach adopted by Sadiq et al. [27] and Namawejje et al. [28], whereby system (1) is put in the following form:

$$
Y^{\prime}=B Y+G(Y),
$$

where

$$
Y=\left(S_{H}, I_{H l}, I_{H h}, T_{H}, S_{A}, I_{A l}, I_{A h}, L_{E}, F_{E}\right)^{T} .
$$

$B$ is the $9 \times 9$ matrix

$$
G(Y)=\left[\begin{array}{c}
b_{H} N_{H}-\left(1-w_{5}(t)\right) \psi_{E H} S_{H}-\left(1-w_{4}(t)\right) \psi_{A H} S_{H} \\
\left(1-w_{4}(t)\right) \psi_{A H} S_{H}-\left(1-w_{5}(t)\right) \psi_{E H} I_{H l} \\
\left(1-w_{5}(t)\right) \psi_{E H} S_{H}+\left(1-w_{5}(t)\right) \psi_{E H} I_{H l} \\
0 \\
b_{A} N_{A}-\left(1-w_{2}(t)\right) \psi_{A A} S_{A}-\left(1-w_{3}(t)\right) \psi_{E A} S_{A} \\
\left(1-w_{2}(t)\right) \psi_{A A} S_{A}-\left(1-w_{3}(t)\right) \psi_{E A} I_{A l} \\
\left(1-w_{3}(t)\right) \psi_{E A} S_{A}+\left(1-w_{3}(t)\right) \psi_{E A} I_{A l} \\
0 \\
0
\end{array}\right]
$$


B

$$
=\left[\begin{array}{ccccccccc}
-\mu_{H} & 0 & 0 & \omega & 0 & 0 & 0 & 0 & 0 \\
0 & -\Psi_{1} & 0 & 0 & 0 & 0 & 0 & 0 & 0 \\
0 & 0 & -\Psi_{2} & 0 & 0 & 0 & 0 & 0 & 0 \\
0 & p_{1} & p_{2} & -\Psi_{3} & 0 & 0 & 0 & 0 & 0 \\
0 & 0 & 0 & 0 & -\mu_{A} & 0 & 0 & 0 & 0 \\
0 & 0 & 0 & 0 & 0 & -\mu_{A} & 0 & 0 & 0 \\
0 & 0 & 0 & 0 & 0 & 0 & -\left(\mu_{A}+\sigma_{A}\right) & 0 & 0 \\
0 & 0 & \delta_{e} & 0 & 0 & 0 & \delta_{e} & -\Psi_{5} & 0 \\
0 & 0 & 0 & 0 & 0 & 0 & \Psi_{4} & \gamma_{L} & -\Psi_{6}
\end{array}\right],
$$

where

$$
\begin{aligned}
& \Psi_{1}=\left(p_{1}+\mu_{H}\right), \\
& \Psi_{2}=\left(p_{2}+\mu_{H}+\sigma_{H}\right), \\
& \Psi_{3}=\left(\omega+\mu_{H}\right), \\
& \Psi_{4}=\left(1-w_{2}\right) \varepsilon_{A}, \\
& \Psi_{5}=\left\{\frac{\delta_{e}}{K}\left(I_{H h}+I_{A h}\right)+\left(\gamma_{L}+\mu_{L}\right)\right\}, \\
& \Psi_{6}=\left(\mu_{F}+w_{1}+\frac{r_{F}}{k+F_{E}}\right)
\end{aligned}
$$

and $Y^{\prime}$ denotes the derivative of $Y$ with respect to time $t$. System (4) is a nonlinear system with a bounded coefficient. We set

$$
R(Y)=B Y+G(Y)
$$

The second term on the right-hand side of $(7 \mathrm{~d}), G(Y)$, satisfies

$$
\begin{aligned}
\mid G & \left(Y_{1}\right)-G\left(Y_{2}\right) \mid \leq H_{1}\left(\left|S_{1 H}(t)-S_{2 H}(t)\right|\right) \\
& +H_{2}\left(\left|I_{1 H l}(t)-I_{2 H l}(t)\right|\right) \\
& +H_{3}\left(\left|I_{1 H h}(t)-I_{2 H h}(t)\right|\right) \\
& +H_{4}\left(\left|T_{1 H}(t)-T_{2 H}(t)\right|\right)+H_{5}\left(\left|S_{1 A}(t)-S_{2 A}(t)\right|\right) \\
& +H_{6}\left(\left|I_{1 A l}(t)-I_{2 A l}(t)\right|\right)+H_{7}\left(\left|I_{1 A h}(t)-I_{2 A h}(t)\right|\right) \\
& +H_{8}\left(\left|L_{1 E}(t)-L_{2 E}(t)\right|\right)+H_{9}\left(\left|F_{1 E}(t)-F_{2 E}(t)\right|\right) \\
& \leq H\left(\left(\left|S_{1 H}(t)-S_{2 H}(t)\right|\right)+\left(\left|I_{1 H l}(t)-I_{2 H l}(t)\right|\right)\right. \\
& +\left(\left|I_{1 H h}(t)-I_{2 H h}(t)\right|\right)+\left(\left|T_{1 H}(t)-T_{2 H}(t)\right|\right) \\
& +\left(\left|S_{1 A}(t)-S_{2 A}(t)\right|\right)+\left(\left|I_{1 A l}(t)-I_{2 A l}(t)\right|\right) \\
& +\left(\left|I_{1 A h}(t)-I_{2 A h}(t)\right|\right)+\left(\left|L_{1 E}(t)-L_{2 E}(t)\right|\right) \\
& \left.-\left(\left|F_{1 E}(t)+F_{2 E}(t)\right|\right)\right),
\end{aligned}
$$

where the positive constant $H=\max \left(H_{i}\right.$ for $\left.i=1, \ldots, 9\right)$ is independent of the state variables. Also we have $\mid R\left(Y_{1}\right)-$ $R\left(Y_{2}\right)|\leq H| Y_{1}-Y_{2} \mid$, where $H=\sum_{i=1}^{9} H_{i}+\|M\|<\infty$. So, it follows that the function $R$ is uniformly Lipchitz continuous.
From the definition of control variables and nonnegative initial conditions we can see that a solution of the system (1) exists [29].

The integrand in the objective functional (4) which is given by the following equation $L(t, y, w)=R_{1} I_{H h}+R_{2} I_{H l}+$ $R_{3} I_{A h}+R_{4} I_{A l}+R_{5} F_{E}+(1 / 2) \sum_{i=1}^{5} Z_{i} w_{i}^{2}$ is convex in the control set $\Pi$.

We must verify the condition that there exist a constant $\omega>1$ and positive numbers $\tau_{1}$ and $\tau_{2}$ such that

$$
\begin{aligned}
& L(t, y, w) \geq \tau_{1}\left(\sum_{i=1}^{5}\left|w_{i}\right|^{2}\right)^{\omega / 2}-\tau_{2}, \\
& R_{1} I_{H h}+R_{2} I_{H l}+R_{3} I_{A h}+R_{4} I_{A l}+R_{5} F_{E}+\frac{1}{2} \sum_{i=1}^{5} Z_{i} w_{i}^{2} \\
& \quad \geq \frac{1}{2} \sum_{i=1}^{5} Z_{i} w_{i}^{2} \\
& \frac{1}{2} \sum_{i=1}^{5} Z_{i} w_{i}^{2} \geq \tau_{1}\left(\sum_{i=1}^{5}\left|w_{i}\right|^{2}\right)^{\Phi / 2}-\tau_{2} .
\end{aligned}
$$

The last condition is satisfied when $\omega=2, \tau_{2}>0$, and $\tau_{1}=$ $\min \left\{Z_{1}, Z_{2}, Z_{3}, Z_{4}, Z_{5}\right\}$. This ends the proof.

3.2. Determination of the Necessary Conditions for Optimality. The necessary conditions include the optimality solutions and the adjoint equations that an optimal control must satisfy which come from Pontryagin's maximum principle (Pontryagin's et al., 1962). This principle converts systems (1) and (4) into a problem of minimizing pointwise Hamiltonian function $H$, which is formed by allowing each of the adjoint variables to correspond to each of the state variables accordingly and combining the results with the objective functional [21]. The resulting equation is as given by

$$
\begin{aligned}
H(t, y, w, \lambda)= & R_{1} I_{H h}+R_{2} I_{H l}+R_{3} I_{A h}+R_{4} I_{A l} \\
& +R_{5} F_{E}+\frac{1}{2} \sum_{i=1}^{5} Z_{i} w_{i}^{2}+\sum_{i=1}^{9} \lambda_{i} Q_{i},
\end{aligned}
$$

where

$\lambda_{i}$ for $i=1, \ldots, 9$ are the adjoint functions associated with the state equations in (1),

$Q_{i}$ for $i=1, \ldots, 9$ is the right-hand side of the differential equations of $i$ th state variable in system (1).

The expanded form of Hamiltonian function in (11) is given by

$$
\begin{gathered}
H(t, y, w, \lambda)=R_{1} I_{H h}+R_{2} I_{H l}+R_{3} I_{A h}+R_{4} I_{A l} \\
+R_{5} F_{E}+\frac{1}{2} \sum_{i=1}^{5} Z_{i} w_{i}^{2}+\lambda_{1}\left\{b_{H} N_{H}+\omega T_{H}\right.
\end{gathered}
$$




$$
\begin{aligned}
& \left.-\left(1-w_{5}\right) \psi_{E H} S_{H}-\left(1-w_{4}\right) \psi_{A H} S_{H}-\mu_{H} S_{H}\right\} \\
& +\lambda_{2}\left\{\left(1-w_{4}\right) \psi_{A H} S_{H}-\left(1-w_{5}\right) \psi_{E H} I_{H l}-p_{1} I_{H l}\right. \\
& \left.-\mu_{H} I_{H l}\right\}+\lambda_{3}\left\{\left(1-w_{5}\right) \psi_{E H} S_{H}+\left(1-w_{5}\right) \psi_{E H} I_{H l}\right. \\
& \left.-p_{2} I_{H h}-\left(\mu_{H}+\sigma_{H}\right) I_{H h}\right\}+\lambda_{4}\left\{p_{1} I_{H l}+p_{2} I_{H h}\right. \\
& \left.-\omega T_{H}-\mu_{H} T_{H}\right\}+\lambda_{5}\left\{b_{A} N_{A}-\left(1-w_{2}\right) \psi_{A A} S_{A}\right. \\
& \left.-\left(1-w_{3}\right) \psi_{E A} S_{A}-\mu_{A} S_{A}\right\}+\lambda_{6}\left\{\left(1-w_{2}\right) \psi_{A A} S_{A}\right. \\
& \left.-\left(1-w_{3}\right) \psi_{E A} I_{A l}-\mu_{A} I_{A l}\right\}+\lambda_{7}\left\{\left(1-w_{3}\right) \psi_{E A} S_{A}\right. \\
& \left.+\left(1-w_{3}\right) \psi_{E A} I_{A l}-\left(\mu_{A}+\sigma_{A}\right) I_{A h}\right\} \\
& +\lambda_{8}\left\{\delta_{e}\left(1-\frac{L_{E}}{K}\right)\left(I_{H h}+I_{A h}\right)-\left(\gamma_{L}+\mu_{L}\right) L_{E}\right\} \\
& +\lambda_{9}\left\{\gamma_{L} L_{E}+\left(1-w_{2}\right) \varepsilon_{A} I_{A h}-\left(\mu_{F}+w_{1}\right) F_{E}\right. \\
& \left.-\frac{r_{F} F_{E}}{\left(k+F_{E}\right)}\right\} .
\end{aligned}
$$

The optimality equations are obtained when taking the partial derivative of the Hamiltonian function $H$ with respect to the control variables $\left(w_{1}, w_{2}, w_{3}, w_{4}, w_{5}\right)$, respectively, and the time derivative of adjoint equation $\lambda^{\prime}$ which is obtained by taking the negative partial derivative of $H$ with respect to the model state variables $y(t)$ such that $\lambda^{\prime}=-H_{y}$.

Theorem 2. There exist an optimal control set $\left(w_{1}^{*}, w_{2}^{*}, w_{3}^{*}\right.$, $\left.w_{4}^{*}, w_{5}^{*}\right)$ and their corresponding state solutions $S_{H}^{*}, I_{H l}^{*}, I_{H h}^{*}$, $T_{H}^{*}, S_{A}^{*}, I_{A l}^{*}, I_{A h}^{*}, L_{E}^{*}$, and $F_{E}^{*}$ that minimize $J\left(w_{1}, w_{2}, w_{3}\right.$, $\left.w_{4}, w_{5}\right)$, and therefore there exist adjoint functions $\lambda_{1}, \lambda_{2}, \ldots, \lambda_{9}$ such that

$$
\begin{aligned}
& \frac{d \lambda_{1}}{d t} \\
& =\lambda_{1} \mu_{H}-\left(1-w_{4}(t)\right)\left(\lambda_{2}-\lambda_{1}\right) \psi_{A H} \\
& \quad-\left(1-w_{5}(t)\right)\left(\lambda_{3}-\lambda_{1}\right) \psi_{E H}
\end{aligned}
$$

$\frac{d \lambda_{2}}{d t}$

$$
\begin{aligned}
= & \left(1-w_{5}(t)\right) \psi_{E H}\left(\lambda_{2}-\lambda_{3}\right)+\lambda_{2}\left(p_{1}+\mu_{H}\right) \\
& -\lambda_{4} p_{1}-R_{2}
\end{aligned}
$$

$$
\begin{aligned}
& \frac{d \lambda_{3}}{d t} \\
& =\lambda_{3}\left(p_{2}+\mu_{H}+\sigma_{H}\right)-\lambda_{8} \delta_{e}\left(1-\frac{L_{E}}{K}\right)-\lambda_{4} p_{2} \\
& -R_{1} \\
& \frac{d \lambda_{4}}{d t}=\lambda_{4}\left(\omega+\mu_{H}\right)-\lambda_{1} \omega \\
& \frac{d \lambda_{5}}{d t} \\
& =\lambda_{5} \mu_{A}-\left(1-w_{2}(t)\right)\left(\lambda_{6}-\lambda_{5}\right) \psi_{A A} \\
& -\left(1-w_{3}(t)\right)\left(\lambda_{7}-\lambda_{5}\right) \psi_{E A} \\
& \frac{d \lambda_{6}}{d t}=\left(\lambda_{6}-\lambda_{7}\right)\left(1-w_{3}(t)\right) \psi_{E A}+\lambda_{6} \mu_{A}-R_{4} \\
& \frac{d \lambda_{7}}{d t} \\
& =\lambda_{7}\left(\mu_{A}+\sigma_{A}\right)-\frac{\left(1-w_{4}(t)\right)\left(\lambda_{2}-\lambda_{1}\right) \rho_{A H} S_{H}}{N_{H}} \\
& -\lambda_{8} \delta_{e}\left(1-\frac{L_{E}}{K}\right) \\
& -\left\{\lambda_{9} \varepsilon_{A}+\frac{\left(\lambda_{6}-\lambda_{5}\right) \rho_{A} S_{A}}{N_{A}}\right\}\left(1-w_{2}(t)\right)-R_{3} \\
& \frac{d \lambda_{8}}{d t}=\frac{\lambda_{8}\left(I_{H h}+I_{A h}\right) \delta_{e}}{K}+\lambda_{8}\left(\gamma_{L}+\mu_{L}\right)-\lambda_{9} \gamma_{L} \\
& \frac{d \lambda_{9}}{d t} \\
& =\lambda_{9}\left\{\left(\mu_{F}+w_{1}(t)\right)+\frac{r_{F} k}{\left(k+F_{E}\right)^{2}}\right\}-R_{5} \\
& -\chi_{\mathrm{H}}\left(1-w_{5}(t)\right)\left\{S_{H}\left(\lambda_{3}-\lambda_{1}\right)+I_{H l}\left(\lambda_{3}-\lambda_{2}\right)\right\} \\
& -\chi_{\mathrm{A}}\left(1-w_{3}(t)\right)\left\{S_{A}\left(\lambda_{7}-\lambda_{5}\right)+I_{A l}\left(\lambda_{7}-\lambda_{6}\right)\right\} \text {, }
\end{aligned}
$$

where $\chi_{H}=\alpha_{E H} \beta_{E H} r_{F} k /\left(k+F_{E}\right)^{2}$ and $\chi_{A}=\alpha_{E A} \beta_{E A} r_{F} k /$ $\left(k+F_{E}\right)^{2}$ with transversality conditions, $\left\{\lambda_{i}(T)\right.$ for $i=$ $1,2, \ldots, 9\}=0$, and the control variables $\left(w_{1}^{*}, w_{2}^{*}, w_{3}^{*}, w_{4}^{*}, w_{5}^{*}\right)$ satisfy the following optimality conditions:

$$
\begin{aligned}
& w_{1}^{*}=\min \quad\left\{\max \left\{0, \frac{\lambda_{9} F_{E}}{Z_{1}}\right\}, 1\right\}, \\
& w_{2}^{*}=\min \left\{\max \left\{0, \frac{\left\{\psi_{A A} S_{A}\left(\lambda_{6}-\lambda_{5}\right)+\lambda_{9} \varepsilon_{A} I_{A h}\right\}}{Z_{2}}\right\}, 1\right\}, \\
& w_{3}^{*}=\min \quad\left\{\max \left\{0, \frac{\psi_{E A}\left\{S_{A}\left(\lambda_{7}-\lambda_{5}\right)+I_{A l}\left(\lambda_{7}-\lambda_{6}\right)\right\}}{Z_{3}}\right\}, 1\right\},
\end{aligned}
$$




$$
\begin{aligned}
& w_{4}^{*}=\min \quad\left\{\max \left\{0, \frac{\psi_{A H} S_{H}\left(\lambda_{2}-\lambda_{1}\right)}{Z_{4}}\right\}, 1\right\}, \\
& w_{5}^{*}=\min \left\{\max \left\{0, \frac{\psi_{E H}\left\{S_{H}\left(\lambda_{3}-\lambda_{1}\right)+I_{H l}\left(\lambda_{3}-\lambda_{2}\right)\right\}}{Z_{5}}\right\}, 1\right\} .
\end{aligned}
$$

Proof. The differential equations for the adjoints are standard results from Pontryagin's maximum principle (1962). Given the Hamiltonian function in (12) the adjoint equations can be easily computed by

$$
\begin{aligned}
& \frac{d \lambda_{1}}{d t}=-\frac{d H}{d S_{H}}, \\
& \frac{d \lambda_{2}}{d t}=-\frac{d H}{d I_{H l}}, \\
& \frac{d \lambda_{3}}{d t}=-\frac{d H}{d I_{H h}}, \\
& \frac{d \lambda_{4}}{d t}=-\frac{d H}{d T_{H}}, \\
& \frac{d \lambda_{5}}{d t}=-\frac{d H}{d S_{A}} \\
& \frac{d \lambda_{6}}{d t}=-\frac{d H}{d I_{A l}}, \\
& \frac{d \lambda_{7}}{d t}=-\frac{d H}{d I_{A h}}, \\
& \frac{d \lambda_{8}}{d t}=-\frac{d H}{d L_{E}}, \\
& \frac{d \lambda_{9}}{d t}=-\frac{d H}{d F_{E}} .
\end{aligned}
$$

Therefore, the adjoint system evaluated at optimal controls $w_{1}, w_{2}, w_{3}, w_{4}$, and $w_{5}$ and the corresponding model state variables $S_{H}, I_{H l}, I_{H h}, T_{H}, S_{A}, I_{A l}, I_{A h}, L_{E}, F_{E}$ is as given by

$$
\begin{aligned}
& \frac{d \lambda_{1}}{d t} \\
& =\lambda_{1} \mu_{H}-\left(1-w_{4}(t)\right)\left(\lambda_{2}-\lambda_{1}\right) \psi_{A H} \\
& \quad-\left(1-w_{5}(t)\right)\left(\lambda_{3}-\lambda_{1}\right) \psi_{E H} \\
& \frac{d \lambda_{2}}{d t} \quad\left(1-w_{5}(t)\right) \psi_{E H}\left(\lambda_{2}-\lambda_{3}\right)+\lambda_{2}\left(p_{1}+\mu_{H}\right) \\
& \quad-\lambda_{4} p_{1}-R_{2} \\
& \frac{d \lambda_{3}}{d t} \\
& =\lambda_{3}\left(p_{2}+\mu_{H}+\sigma_{H}\right)-\lambda_{8} \delta_{e}\left(1-\frac{L_{E}}{K}\right)-\lambda_{4} p_{2}
\end{aligned}
$$

$$
-R_{1}
$$$$
\frac{d \lambda_{4}}{d t}=\lambda_{4}\left(\omega+\mu_{H}\right)-\lambda_{1} \omega
$$$$
\frac{d \lambda_{5}}{d t}
$$$$
=\lambda_{5} \mu_{A}-\left(1-w_{2}(t)\right)\left(\lambda_{6}-\lambda_{5}\right) \psi_{A A}
$$$$
-\left(1-w_{3}(t)\right)\left(\lambda_{7}-\lambda_{5}\right) \psi_{E A}
$$$$
\frac{d \lambda_{6}}{d t}=\left(\lambda_{6}-\lambda_{7}\right)\left(1-w_{3}(t)\right) \psi_{E A}+\lambda_{6} \mu_{A}-R_{4}
$$$$
\frac{d \lambda_{7}}{d t}
$$

$$
\begin{aligned}
= & \lambda_{7}\left(\mu_{A}+\sigma_{A}\right)-\frac{\left(1-w_{4}(t)\right)\left(\lambda_{2}-\lambda_{1}\right) \rho_{A H} S_{H}}{N_{H}} \\
& -\lambda_{8} \delta_{e}\left(1-\frac{L_{E}}{K}\right) \\
& -\left\{\lambda_{9} \varepsilon_{A}+\frac{\left(\lambda_{6}-\lambda_{5}\right) \rho_{A} S_{A}}{N_{A}}\right\}\left(1-w_{2}(t)\right)-R_{3}
\end{aligned}
$$$$
\frac{d \lambda_{8}}{d t}=\frac{\lambda_{8}\left(I_{H h}+I_{A h}\right) \delta_{e}}{K}+\lambda_{8}\left(\gamma_{L}+\mu_{L}\right)-\lambda_{9} \gamma_{L}
$$

$\frac{d \lambda_{9}}{d t}$

$$
\begin{aligned}
= & \lambda_{9}\left\{\left(\mu_{F}+w_{1}(t)\right)+\frac{r_{F} k}{\left(k+F_{E}\right)^{2}}\right\}-R_{5} \\
& -\chi_{H}\left(1-w_{5}(t)\right)\left\{S_{H}\left(\lambda_{3}-\lambda_{1}\right)+I_{H l}\left(\lambda_{3}-\lambda_{2}\right)\right\} \\
& -\chi_{A}\left(1-w_{3}(t)\right)\left\{S_{A}\left(\lambda_{7}-\lambda_{5}\right)+I_{A l}\left(\lambda_{7}-\lambda_{6}\right)\right\},
\end{aligned}
$$

where $\chi_{H}=\alpha_{E H} \beta_{E H} r_{F} k /\left(k+F_{E}\right)^{2}$ and $\chi_{A}=\alpha_{E A} \beta_{E A} r_{F} k /$ $\left(k+F_{E}\right)^{2}$ with transversality conditions, $\left\{\lambda_{i}(T)\right.$ for $i=$ $1,2, \ldots, 9\}=0$, and the characterization of optimal controls $w_{1}^{*}, w_{2}^{*}, w_{3}^{*}, w_{4}^{*}, w_{5}^{*}$; that is, the optimality equations are based on the conditions:

$$
\frac{\partial H}{\partial w_{1}}=\frac{\partial H}{\partial w_{2}}=\frac{\partial H}{\partial w_{3}}=\frac{\partial H}{\partial w_{4}}=\frac{\partial H}{\partial w_{5}}=0 .
$$

Subject to (17) the optimality condition given the Lebesgue measurable control set $\Pi=\left\{0 \leq w_{i}(t) \leq 1\right.$, for 
$i=1,2, \ldots, 5$ and $t \in[0, T]\}$, where the control variables $w_{1}, w_{2}, w_{3}, w_{4}, w_{5}$ are measurable functions, is given by

$$
\begin{aligned}
\frac{\partial H}{\partial w_{1}}= & Z_{1} w_{1}-\lambda_{9} F_{E} \\
\frac{\partial H}{\partial w_{2}}= & Z_{2} w_{2}+\lambda_{5} \psi_{A A} S_{A}-\lambda_{6} \psi_{A A} S_{A}-\lambda_{9} \varepsilon_{A} I_{A h} \\
\frac{\partial H}{\partial w_{3}}= & Z_{3} w_{3}+\lambda_{5} \psi_{E A} S_{A}+\lambda_{6} \psi_{E A} I_{A l}-\lambda_{7} \psi_{E A} S_{A} \\
& -\lambda_{7} \psi_{E A} I_{A l} \\
\frac{\partial H}{\partial w_{4}}= & Z_{4} w_{4}+\lambda_{1} \psi_{A H} S_{H}-\lambda_{2} \psi_{A H} S_{H} \\
\frac{\partial H}{\partial w_{5}}= & Z_{5} w_{5}+\lambda_{1} \psi_{E H} S_{H}+\lambda_{2} \psi_{E H} I_{H l}-\lambda_{3} \psi_{E H} S_{H} \\
& -\lambda_{3} \psi_{E H} I_{H l} \cdot
\end{aligned}
$$

If we set $\partial H / \partial w_{i}=0$ at $w_{i}^{*}$ the results are the same as in characterization in (14).

In order to satisfy the given bounds for the control functions (i.e., $0 \leq w_{i} \leq 1$ and $t \in[0, T]$ ) the optimal control is restricted to $w_{i}^{*}=\min \left\{\max \left(0, w_{i}\right), 1\right\}$. Therefore using the bounds for the controls $w_{1}, w_{2}, w_{3}, w_{4}, w_{5}$ we have the following.

The bounds and the impact notation for the control $w_{1}$ are, respectively, given as

$$
\begin{aligned}
& w_{1}^{*}= \begin{cases}\frac{\lambda_{9} F_{E}^{*}}{Z_{1}} & \text { if } 0 \leq \frac{\lambda_{9} F_{E}^{*}}{Z_{1}} \leq 1, \\
0 & \text { if } \frac{\lambda_{9} F_{E}^{*}}{Z_{1}} \leq 0, \\
1 & \text { if } \frac{\lambda_{9} F_{E}^{*}}{Z_{1}} \geq 1,\end{cases} \\
& w_{1}^{*}=\min \left\{\max \left\{0, \frac{\lambda_{9} F_{E}^{*}}{Z_{1}}\right\}, 1\right\} .
\end{aligned}
$$

Similar four-step arguments hold for optimal control schedules $w_{2}^{*}, w_{3}^{*}, w_{4}^{*}$, and $w_{5}^{*}$ in the same way as in (19) based on the characterization in (14).

3.3. The Optimality System. The optimality system consists of the state system and adjoint system with initial and transversal conditions together with characterization of optimal control. Any optimal control pair must satisfy this optimality system as indicated in (20) and (22), respectively.

$$
\begin{aligned}
& \frac{d S_{H}(t)}{d t}=b_{H} N_{H}-(1 \\
& -\min \left\{\max \left\{0, \frac{\psi_{E H}^{*}\left\{S_{H}\left(\lambda_{3}-\lambda_{1}\right)+I_{H l}\left(\lambda_{3}-\lambda_{2}\right)\right\}}{Z_{5}}\right\},\right.
\end{aligned}
$$

$$
\begin{aligned}
& 1\}) \psi_{E H}^{*} S_{H}+\omega T_{H}-(1 \\
& \left.-\min \left\{\max \left\{0, \frac{\psi_{A H}^{*} S_{H}\left(\lambda_{2}-\lambda_{1}\right)}{Z_{4}}\right\}, 1\right\}\right) \psi_{A H}^{*} S_{H} \\
& -\mu_{H} S_{H} \\
& \frac{d I_{H l}(t)}{d t}=\left(1-\min \left\{\max \left\{0, \frac{\psi_{A H}^{*} S_{H}\left(\lambda_{2}-\lambda_{1}\right)}{Z_{4}}\right\}, 1\right\}\right) \\
& \cdot \psi_{A H}^{*} S_{H}-(1 \\
& -\min \left\{\max \left\{0, \frac{\psi_{E H}^{*}\left\{S_{H}\left(\lambda_{3}-\lambda_{1}\right)+I_{H l}\left(\lambda_{3}-\lambda_{2}\right)\right\}}{Z_{5}}\right\},\right. \\
& 1\}) \psi_{E H}^{*} I_{H l}-p_{1} I_{H l}-\mu_{H} I_{H l} \\
& \frac{d I_{H h}(t)}{d t}=(1 \\
& -\min \left\{\max \left\{0, \frac{\psi_{E H}^{*}\left\{S_{H}\left(\lambda_{3}-\lambda_{1}\right)+I_{H l}\left(\lambda_{3}-\lambda_{2}\right)\right\}}{Z_{5}}\right\},\right. \\
& 1\}) \psi_{E H}^{*}\left(S_{H}+I_{H l}\right)-p_{2} I_{H h}-\left(\mu_{H}+\sigma_{H}\right) I_{H h} \\
& \frac{d T_{H}(t)}{d t}=p_{1} I_{H l}+p_{2} I_{H h}-\omega T_{H}-\mu_{H} T_{H} \\
& \frac{d S_{A}(t)}{d t}=b_{A} N_{A}-(1 \\
& \left.-\min \left\{\max \left\{0, \frac{\psi_{A A}^{*} S_{A}\left(\lambda_{6}-\lambda_{5}\right)+\lambda_{9} \varepsilon_{A} I_{A h}}{Z_{2}}\right\}, 1\right\}\right) \\
& \cdot \psi_{A A}^{*} S_{A}-(1 \\
& -\min \left\{\max \left\{0, \frac{\psi_{E A}^{*}\left\{S_{A}\left(\lambda_{7}-\lambda_{5}\right)+I_{A l}\left(\lambda_{7}-\lambda_{6}\right)\right\}}{Z_{3}}\right\},\right. \\
& 1\}) \psi_{E A}^{*} S_{A}-\mu_{A} S_{A} \\
& \frac{d I_{A l}(t)}{d t}=(1 \\
& \left.-\min \left\{\max \left\{0, \frac{\left\{\psi_{A A}^{*} S_{A}\left(\lambda_{6}-\lambda_{5}\right)+\lambda_{9} \varepsilon_{A} I_{A h}\right\}}{Z_{2}}\right\}, 1\right\}\right) \\
& \cdot \psi_{A A}^{*} S_{A}-(1 \\
& -\min \left\{\max \left\{0, \frac{\psi_{E A}^{*}\left\{S_{A}\left(\lambda_{7}-\lambda_{5}\right)+I_{A l}\left(\lambda_{7}-\lambda_{6}\right)\right\}}{Z_{3}}\right\},\right. \\
& 1\}) \psi_{E A}^{*} I_{A l}-\mu_{A} I_{A l} \\
& \frac{d I_{A h}(t)}{d t}=(1
\end{aligned}
$$




$$
\begin{aligned}
& -\min \left\{\max \left\{0, \frac{\psi_{E A}^{*}\left\{S_{A}\left(\lambda_{7}-\lambda_{5}\right)+I_{A l}\left(\lambda_{7}-\lambda_{6}\right)\right\}}{Z_{3}}\right\},\right. \\
& 1\}) \psi_{E A}^{*}\left(S_{A}+I_{A l}\right)-\left(\mu_{A}+\sigma_{A}\right) I_{A h} \\
& \frac{d L_{E}(t)}{d t}=\delta_{e}\left(1-\frac{L_{E}}{K}\right)\left(I_{H h}+I_{A h}\right)-\left(\gamma_{L}+\mu_{L}\right) L_{E} \\
& \frac{d F_{E}(t)}{d t}=\gamma_{L} L_{E}+(1 \\
& \left.-\min \left\{\max \left\{0, \frac{\left\{\psi_{A A}^{*} S_{A}\left(\lambda_{6}-\lambda_{5}\right)+\lambda_{9} \varepsilon_{A} I_{A h}\right\}}{Z_{2}}\right\}, 1\right\}\right) \\
& \cdot \varepsilon_{A} I_{A h}-\left(\mu_{F}+\min \left\{\max \left\{0, \frac{\lambda_{9} F_{E}}{Z_{1}}\right\}, 1\right\}\right) F_{E} \\
& -\frac{r_{F} F_{E}}{\left(k+F_{E}\right)}
\end{aligned}
$$

with initial conditions

$$
\begin{aligned}
& S_{H}(0)>0, \\
& I_{H l}(0) \geq 0, \\
& I_{H h}(0) \geq 0, \\
& T_{H}(0) \geq 0, \\
& S_{A}(0)>0, \\
& I_{A l}(0) \geq 0, \\
& I_{A h}(0) \geq 0, \\
& L_{E}(0) \geq 0, \\
& F_{E}(0)>0, \\
& \frac{d \lambda_{1}}{d t}= \lambda_{1} \mu_{H}-(1 \\
&-\min \left\{\max \left\{0, \frac{\psi_{E A}^{*}\left\{S_{A}\left(\lambda_{7}-\lambda_{5}\right)+I_{A l}\left(\lambda_{7}-\lambda_{6}\right)\right\}}{Z_{3}}\right\},\right. \\
&1\}) \psi_{E H}^{*}\left(\lambda_{2}-\lambda_{3}\right)+\lambda_{2}\left(p_{1}+\mu_{H}\right)-\lambda_{4} p_{1}-R_{2} \\
& \frac{d \lambda_{3}}{d t}=\lambda_{3}\left(p_{2}+\mu_{H}+\sigma_{H}\right)-R_{1}-\lambda_{4} p_{2}-\lambda_{8} \delta_{e}\left(1-\frac{L_{E}}{K}\right) \\
&1\}) \psi_{A H}^{*}\left(\lambda_{2}-\lambda_{1}\right)-(1 \\
&1\}) \psi_{E H}^{*}\left(\lambda_{3}-\lambda_{1}\right) \\
&-\min \left\{\max \left\{0, \frac{\psi_{E H}^{*}\left\{S_{H}\left(\lambda_{3}-\lambda_{1}\right)+I_{H l}\left(\lambda_{3}-\lambda_{2}\right)\right\}}{Z_{5}}\right\},\right.
\end{aligned}
$$

$$
\begin{aligned}
& \frac{d \lambda_{4}}{d t}=\lambda_{4}\left(\omega+\mu_{H}\right)-\lambda_{1} \omega \\
& \frac{d \lambda_{5}}{d t}=\lambda_{5} \mu_{A}-(1 \\
& \left.-\min \left\{\max \left\{0, \frac{\left\{\psi_{A A}^{*} S_{A}\left(\lambda_{6}-\lambda_{5}\right)+\lambda_{9} \varepsilon_{A} I_{A h}\right\}}{Z_{2}}\right\}, 1\right\}\right) \\
& \cdot \psi_{A A}^{*}\left(\lambda_{6}-\lambda_{5}\right)-(1-\min \{\max \{0, \\
& \left.\left.\left.\frac{\psi_{E A}^{*}\left\{S_{A}\left(\lambda_{7}-\lambda_{5}\right)+I_{A l}\left(\lambda_{7}-\lambda_{6}\right)\right\}}{Z_{3}}\right\}, 1\right\}\right) \psi_{E A}^{*}\left(\lambda_{7}-\lambda_{5}\right) \\
& \frac{d \lambda_{6}}{d t}=\left(\lambda_{6}-\lambda_{7}\right)(1 \\
& -\min \left\{\max \left\{0, \frac{\psi_{E A}^{*}\left\{S_{A}\left(\lambda_{7}-\lambda_{5}\right)+I_{A l}\left(\lambda_{7}-\lambda_{6}\right)\right\}}{Z_{3}}\right\},\right. \\
& 1\}) \psi_{E A}+\lambda_{6} \mu_{A}-R_{4} \\
& \frac{d \lambda_{7}}{d t}=\lambda_{7}\left(\mu_{A}+\sigma_{A}\right)-(1 \\
& \left.-\min \left\{\max \left\{0, \frac{\psi_{A H}^{*} S_{H}\left(\lambda_{2}-\lambda_{1}\right)}{Z_{4}}\right\}, 1\right\}\right) \\
& \cdot \frac{\left(\lambda_{2}-\lambda_{1}\right) \rho_{A H} S_{H}}{N_{H}}-\left\{\lambda_{9} \varepsilon_{A}+\frac{\left(\lambda_{6}-\lambda_{5}\right) \rho_{A} S_{A}}{N_{A}}\right\}(1 \\
& \left.-\min \left\{\max \left\{0, \frac{\left\{\psi_{A A}^{*} S_{A}\left(\lambda_{6}-\lambda_{5}\right)+\lambda_{9} \varepsilon_{A} I_{A h}\right\}}{Z_{2}}\right\}, 1\right\}\right) \\
& -\lambda_{8} \delta_{e}\left(1-\frac{L_{E}}{K}\right)-R_{3} \\
& \frac{d \lambda_{8}}{d t}=\frac{\lambda_{8}\left(I_{H h}+I_{A h}\right) \delta_{e}}{K}+\lambda_{8}\left(\gamma_{L}+\mu_{L}\right)-\lambda_{9} \gamma_{L} \\
& \frac{d \lambda_{9}}{d t}=\lambda_{9}\left\{\left(\mu_{F}+\mu_{F}+\min \left\{\max \left(0, \frac{\lambda_{9} F_{E}}{Z_{1}}\right), 1\right\}\right)\right. \\
& \left.+\frac{r_{F} k}{\left(k+F_{E}\right)^{2}}\right\}-R_{5}-\chi_{H}(1 \\
& -\min \left\{\max \left\{0, \frac{\psi_{E H}^{*}\left\{S_{H}^{*}\left(\lambda_{3}-\lambda_{1}\right)+I_{H l}^{*}\left(\lambda_{3}-\lambda_{2}\right)\right\}}{Z_{5}}\right\}\right. \text {, } \\
& 1\})\left\{S_{H}\left(\lambda_{3}-\lambda_{1}\right)+I_{H l}\left(\lambda_{3}-\lambda_{2}\right)\right\}-\chi_{A}(1 \\
& -\min \left\{\max \left\{0, \frac{\psi_{E A}^{*}\left\{S_{A}\left(\lambda_{7}-\lambda_{5}\right)+I_{A l}\left(\lambda_{7}-\lambda_{6}\right)\right\}}{Z_{3}}\right\}\right. \text {, } \\
& 1\})\left\{S_{A}\left(\lambda_{7}-\lambda_{5}\right)+I_{A l}\left(\lambda_{7}-\lambda_{6}\right)\right\} \text {, }
\end{aligned}
$$

where $\chi_{H}=\alpha_{E H} \beta_{E H} r_{F} k /\left(k+F_{E}\right)^{2}$ and $\chi_{A}=\alpha_{E A} \beta_{E A} r_{F} k /$ $\left(k+F_{E}\right)^{2}$ with transversality conditions $\left\{\lambda_{i}(T)\right.$ for $i=$ $1,2, \ldots, 9\}=0$. 
TABLE 1: Parameter values for numerical simulation of optimal control model.

\begin{tabular}{|c|c|c|}
\hline Parameter & Value/range & Source/references \\
\hline $\bar{k}$ & $1 \times 10^{4} \mathrm{cell} / \mathrm{m}^{3}$ & Estimated \\
\hline$K$ & $1 \times 10^{5} \mathrm{cell} / \mathrm{m}^{3}$ & Estimated \\
\hline$\gamma_{L}$ & 0.0105 per dayjsup $/ \dot{i}$ & Estimated \\
\hline$\sigma_{H}$ & 0.011 per day & Estimated \\
\hline$\sigma_{A}$ & 0.037 per day & Estimated \\
\hline$\mu_{H}$ & 0.000045 per day & UNICEF. [14] \\
\hline$\mu_{A}$ & $0.0028(360-3600)^{-1}$ per day & Gaff et al. [15]; Radostits. (2001) \\
\hline$\mu_{F}$ & 0.04 per day & Eisele et al. [16], \\
\hline$r_{F}$ & 0.58 per day & Estimated \\
\hline$\mu_{L}$ & 0.08 per day & Estimated \\
\hline$\beta_{E H}$ & 0.19 per day & Estimated \\
\hline$\beta_{E A}$ & 0.48 per day & Estimated \\
\hline$\rho_{\text {AH }}$ & 0.052 per day & Gaff et al. [15] \\
\hline$\rho_{A}$ & $0.26(0.091-0.9)$ per day & Allerson et al. [17] \\
\hline$b_{H}$ & 0.00011 per day & TP, [18]. \\
\hline$b_{A}$ & 0.022 per day & Gaff et al. [15] \\
\hline$\varepsilon_{A}$ & 0.40 per day & Estimated \\
\hline$\delta_{e}$ & 0.12 per day & Estimated \\
\hline$\alpha_{E H}$ & 0.4 & Estimated \\
\hline$\alpha_{E A}$ & 0.6 & Estimated \\
\hline$p_{1}$ & 0.15 & Estimated \\
\hline$p_{2}$ & 0.15 & Estimated \\
\hline$\omega$ & 0.09 & Estimated \\
\hline
\end{tabular}

Source: Kahuru et al. [19].

\section{Numerical Simulation of the Optimal Control Model, Results, and Discussion}

Sometimes it may not be possible to solve the optimality system analytically; instead numerical methods are used to approximate the solutions and display the results. The optimality system is a two-point boundary problem, because of the initial condition of the state system and the terminal condition of the adjoint system [30]. To solve the optimality system with initial conditions for the states and final time conditions for the adjoints, we use the Runge-Kutta fourthorder procedure which is more accurate and elaborative technique. A Runge-Kutta method is a multiple-step method, where the solution at time $t_{k+1}$ is obtained from a defined set of previous values $t_{n-k}, \ldots, t_{k}$ and $n$ is the number of steps. This method is described in a book by Lenhart and Workman [8] and it is known as forward-backward sweep method. The process begins with an initial guess on the control variable and given initial conditions for states, we approximate solutions for state equations using Runge-Kutta forward sweep method. Given the state solutions from previous step and the final time conditions for adjoints, we approximate solutions for adjoint equations using Runge-Kutta backward sweep method. The value of control variables is updated by averaging the previous value and the new value arising from the control characterization. The process is repeated for forward numerical scheme and updating of the controls until successive values of all states, adjoints, and controls are sufficiently close or converge.

4.1. Numerical Simulation of Optimal Control Model. We conduct numerical simulation in order to investigate the effects of the control strategies on the transmission dynamics of Tungiasis. The simulations are performed using MALAB, and we set time in days. The estimated initial values of model state variables are $S_{H}=1000, I_{H l}=200, I_{H h}=300$, $T_{H}=0, S_{A}=900, I_{A l}=200, I_{A h}=300, L_{E}=10000$, and $F_{E}=4000$ and for the adjoint system we have terminal conditions $\left\{\lambda_{i}(T)\right.$ for $\left.i=1, \ldots, 9\right\}=0$, where we set $T=200$ days. The cost coefficients corresponding to state variables are estimated to be $R_{1}=20, R_{2}=10, R_{3}=15, R_{4}=20$, and $R_{5}=$ 25 . The quadratic cost coefficient corresponding to control measures is as well estimated to be $Z_{1}=50, Z_{2}=60, Z_{3}=80$, $Z_{4}=10$, and $Z_{5}=15$. We use a set of parameter values whose sources are from literature and others are estimated as shown in Table 1.

We then plot the graphs to show the effects of the control measures when implemented under different combination options. We first illustrate the situation when no optimal control strategy is implemented as shown in Figures 2(a)-2(d) and then we suggest seven control strategies with different combinations of control measures and compare their performance in order to determine the best option to control the disease for maximum benefit. 

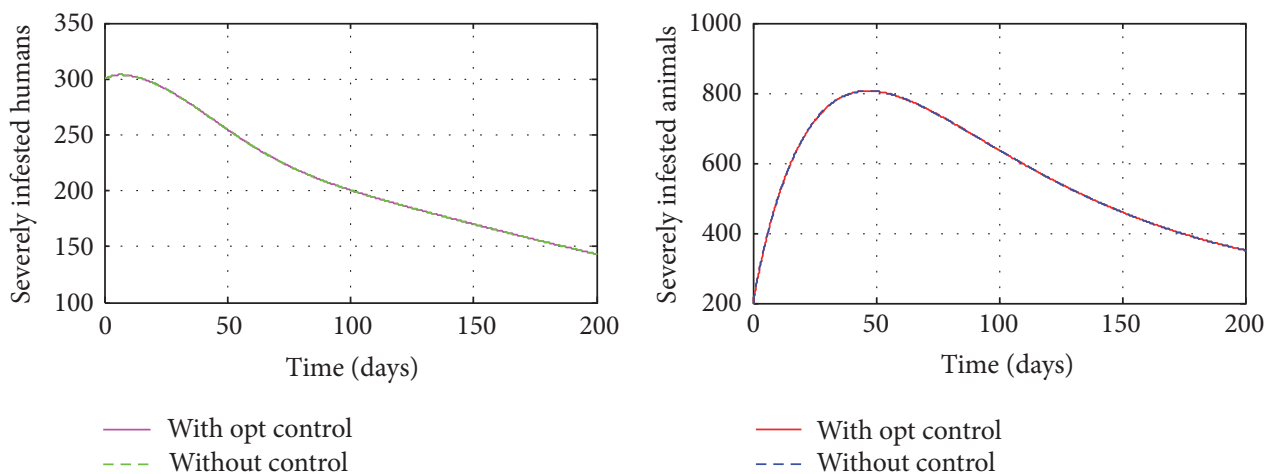

(b)
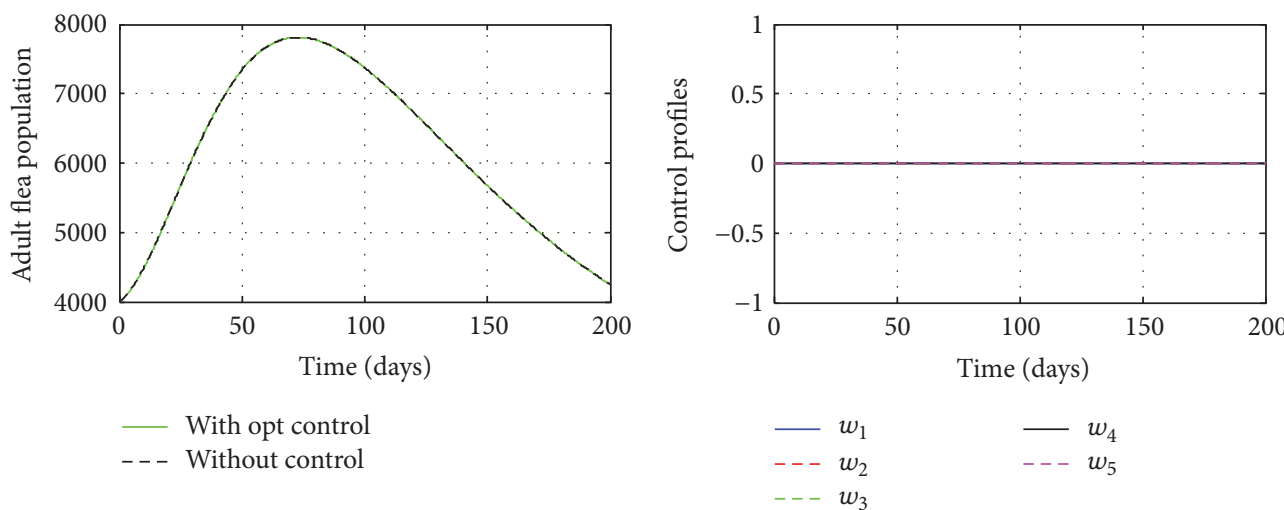

(c)

(d)

FIgURE 2: Simulations of model variables $I_{H h}, I_{A h}, F_{E}$ without intervention.

4.2. Discussion of the Results. In this section, we present the results of the numerical simulation of our optimal control problem by discussing the implications of implementing the seven optimal control strategies on the Tungiasis dynamical model. To observe the effects of the optimal control strategies, we plot the results from simulation of the uncontrolled system and that from the controlled system together in Figures 3-9. To compare the effects of these options, we plot the results together in Figures $10(\mathrm{a})-10(\mathrm{c})$. Thus we consider the following seven combination options.

\subsubsection{When All the Control Strategies Are Not Implemented} (i.e., $w_{1}, w_{2}, w_{3}, w_{4}, w_{5}=0$ ). Figures $2(\mathrm{a})-2(\mathrm{c})$ show the situation where no control strategy is implemented to the dynamical system whereby the model trajectories represented by a dotted lines for severely infested humans, severely infested animal reservoir, and flea populations remain unchanged and the control profiles in Figure 2(d) show that all control measures are at the lower bound (i.e., $w_{1}, w_{2}, w_{3}, w_{4}, w_{5}=0$ ).

4.2.2. Strategy 1: All Control Measures Are Implemented (i.e., $\left.w_{1}, w_{2}, w_{3}, w_{4}, w_{5} \neq 0\right)$. Under strategy 1 , all the control measures $\left(w_{1}, w_{2}, w_{3}, w_{4}, w_{5}\right)$ are used to optimize the objective functional $J(w)$. In Figure 3(d), the control measure $w_{5}$ is at upper bound at the beginning and after 40 days it gradually drops to the lower bound at the final time. The control measure $w_{3}$ is at the upper bound at the beginning and after 45 days it gradually drops to the lower bound at the final time. The control measure $w_{1}$ is at the upper bound at the beginning and after 60 days it drops to the lower bound at the final time. The control measure $w_{4}$ is at the upper bound at the beginning and after 140 days it rapidly drops to the lower bound at the final time and the control measure $w_{2}$ starts at the upper bound at the beginning and remains there until it drops to the lower bound.

4.2.3. Strategy 2: The Control Measures (i.e., $w_{2}, w_{3}, w_{4}, w_{5} \neq$ 0 and $w_{1}=0$ ). Under strategy 2 , the control measures $\left(w_{2}, w_{3}, w_{4}, w_{5}\right)$ are used to optimize the objective functional $J(w)$. In Figure $4(\mathrm{~d})$, the control measure $w_{4}$ is at upper bound at the beginning and after 140 days it rapidly drops to the lower bound at the final time. The control measure $w_{5}$ is at the upper bound at the beginning and after 175 days it rapidly drops to the lower bound at the final time. The control measure $w_{3}$ is at the upper bound at the beginning and after 195 days it drops to the lower bound at the final time. The control measure $w_{2}$ starts at the upper bound at the beginning and remains there until it drops to the lower bound at the final time. The control measure $w_{1}$ is at the lower bound at the beginning and remains there till the final time.

4.2.4. Strategy 3: The Control Measures (i.e., $w_{3}, w_{4}, w_{5} \neq 0$ and $\left.w_{1}, w_{2}=0\right)$. Under strategy 3 , the control measures $\left(w_{3}, w_{4}, w_{5}\right)$ are used to optimize the objective functional 

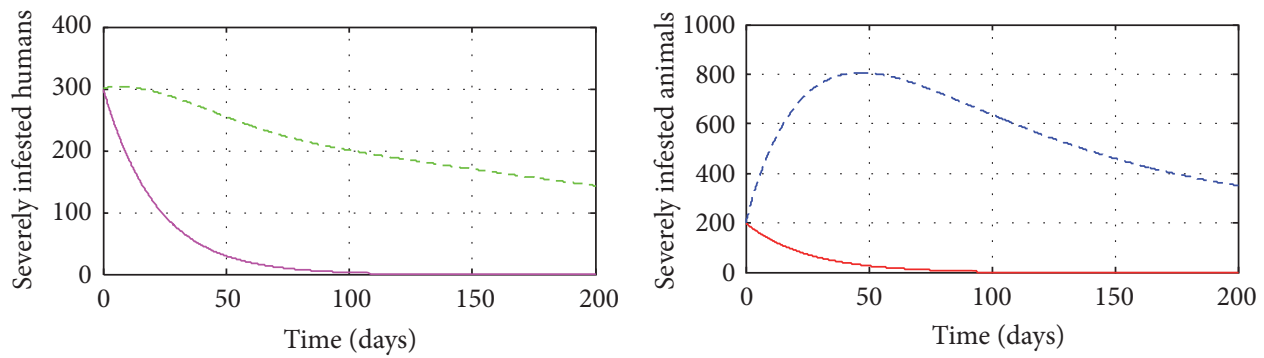

With opt control

- - - Without control

- With opt control
- - Without control

(a)

(b)
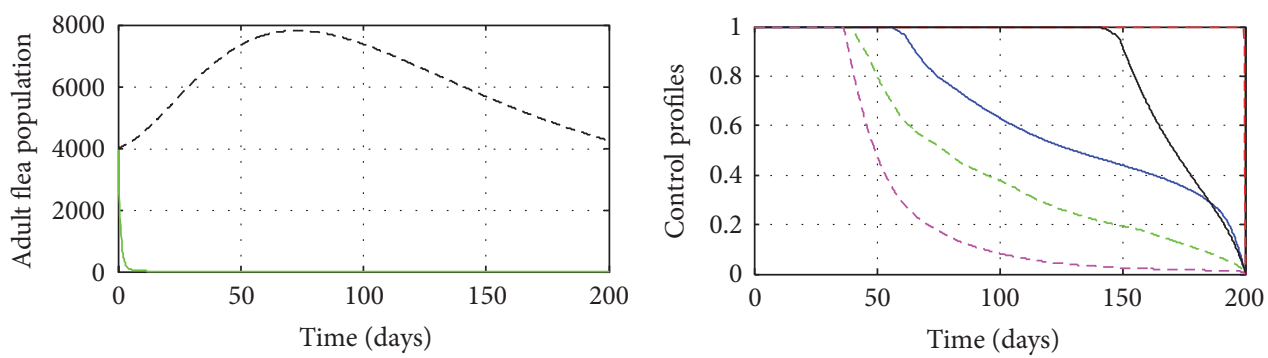

With opt control

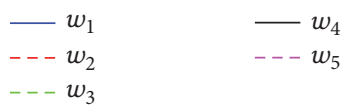

(c)

(d)

FIGURE 3: Optimal solutions for model variables $I_{H h}, I_{A h}, F_{E}$ and the control profiles for $w_{1}, w_{2}, w_{3}, w_{4}, w_{5}$ with $\left(w_{1}, w_{2}, w_{3}, w_{4}, w_{5} \neq 0\right)$.

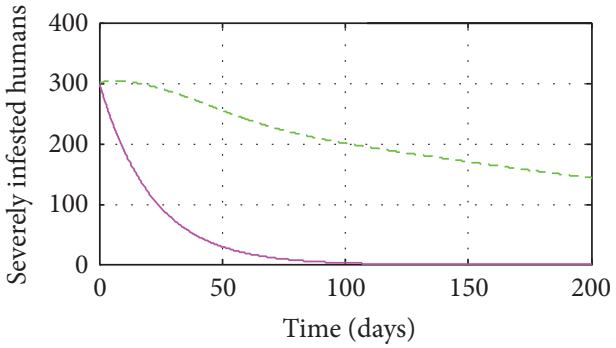

- With opt control

- - - Without control

(a)

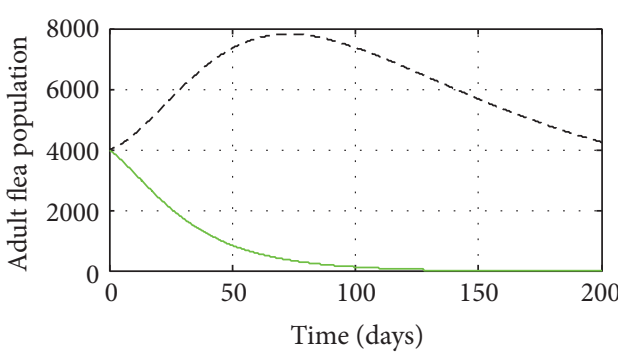

With opt control

- - - Without control

(c)

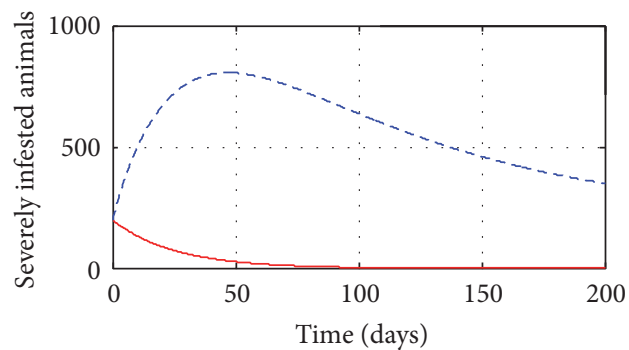

- With opt control

- - - Without control

(b)

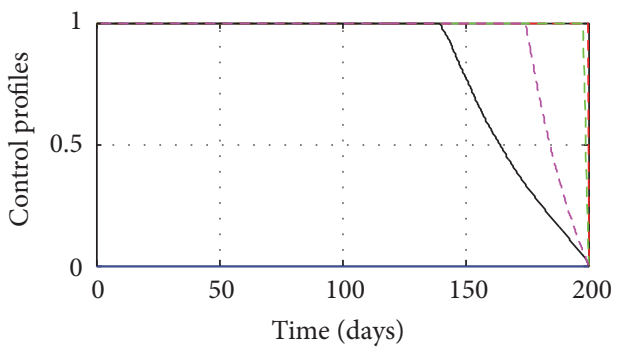

(d)

FIgURE 4: Optimal solutions for model variables $I_{H h}, I_{A h}, F_{E}$ and the control profiles for $w_{1}, w_{2}, w_{3}, w_{4}, w_{5}$ with $\left(w_{2}, w_{3}, w_{4}, w_{5} \neq 0\right)$. 

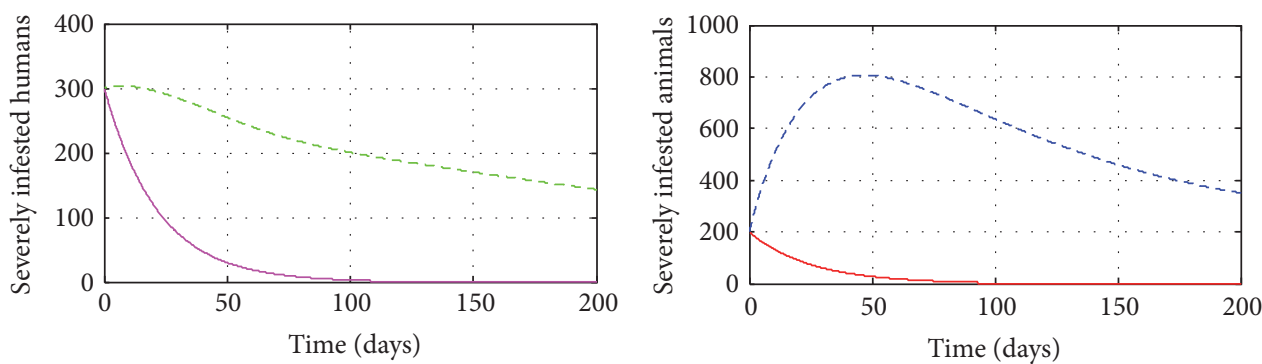

- With opt control

Without control

With opt control

(a)

(b)
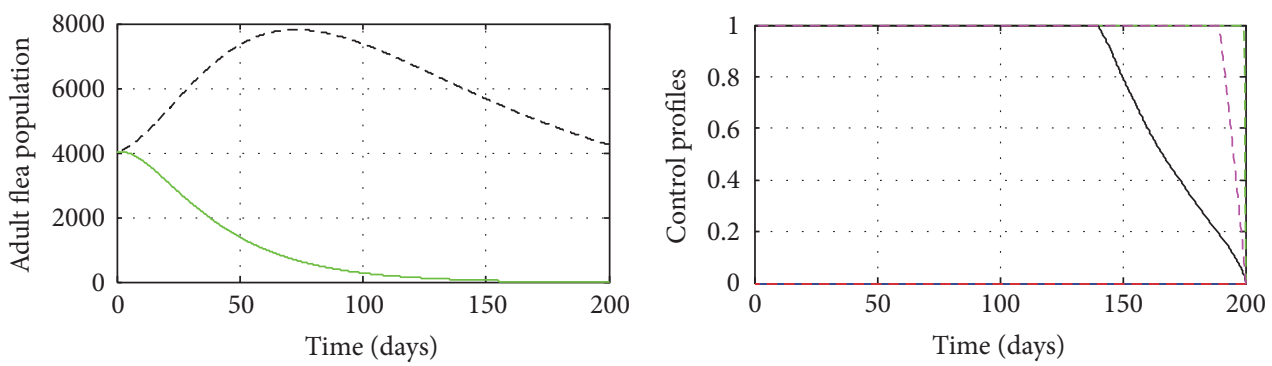

With opt contro

- . - Without control

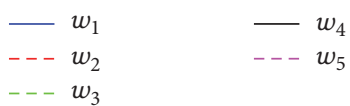

(c)

(d)

Figure 5: Optimal solutions for model variables $I_{H h}, I_{A h}, F_{E}$ and the control profiles for $w_{1}, w_{2}, w_{3}, w_{4}, w_{5}$ with $\left(w_{3}, w_{4}, w_{5} \neq 0\right)$.
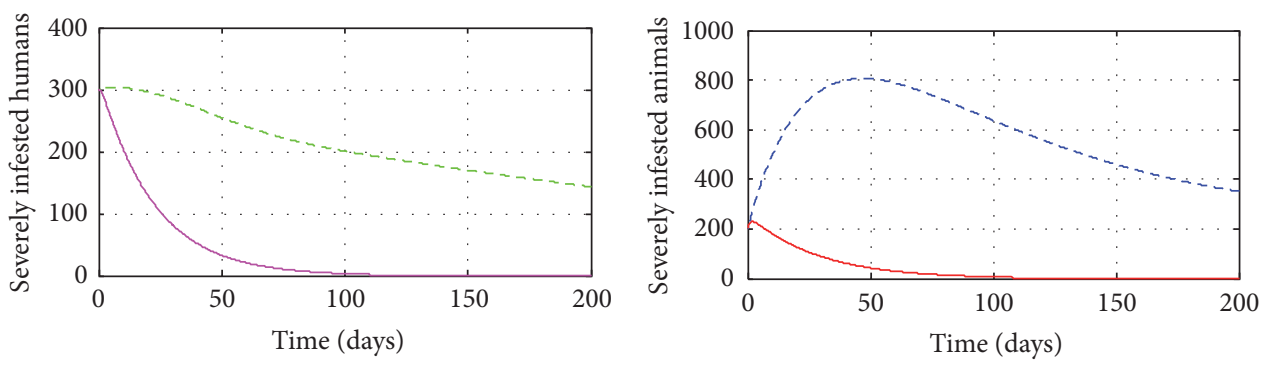

- With opt control

Without control

- With opt control

- - - Without control

(a)

(b)
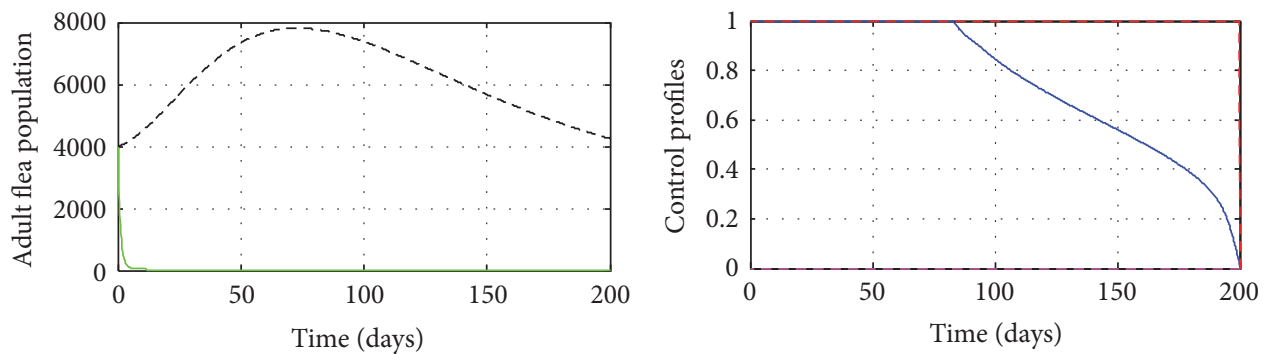

With opt control

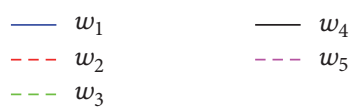

(d)

(c)

Figure 6: Optimal solutions for model variables $I_{H h}, I_{A h}, F_{E}$ and the control profiles for $w_{1}, w_{2}, w_{3}, w_{4}, w_{5}$ with $\left(w_{1}, w_{2} \neq 0\right)$. 

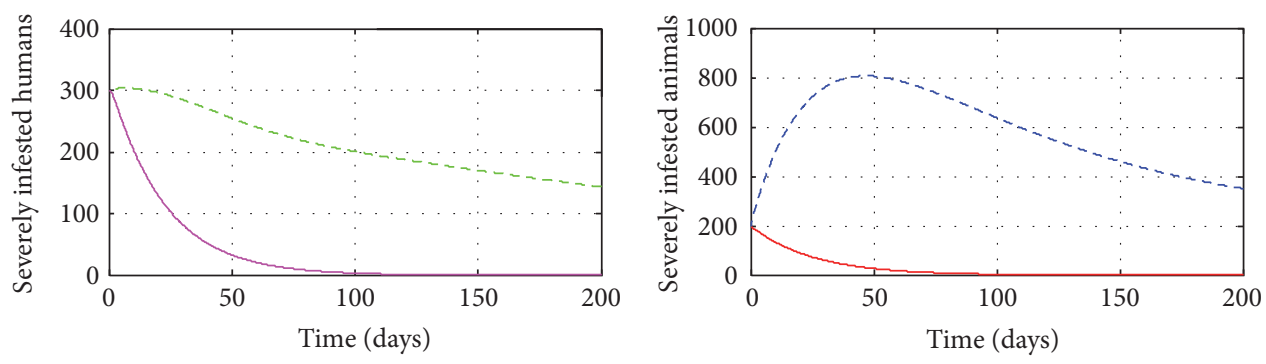

With opt control

Without control

(a)

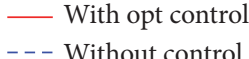

- - - Without control

(b)
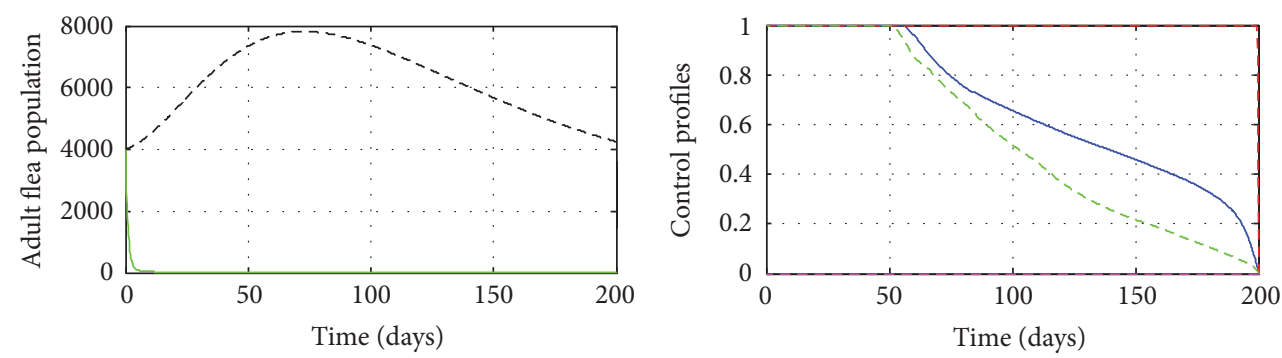

_ With opt control

--- Without control

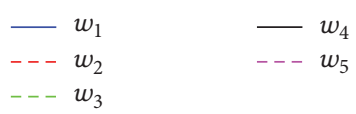

(c)

(d)

FIGURE 7: Optimal solutions for model variables $I_{H h}, I_{A h}, F_{E}$ and the control profiles for $w_{1}, w_{2}, w_{3}, w_{4}, w_{5}$ with $\left(w_{1}, w_{2}, w_{3} \neq 0\right)$.

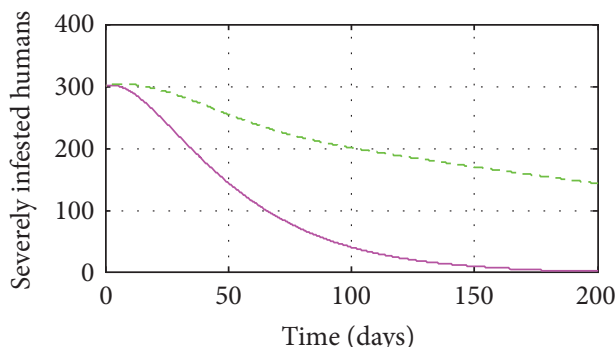

- With opt control Without control

(a)

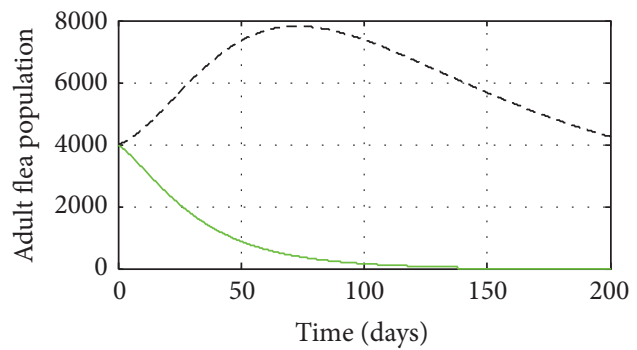

With opt control

- - - Without control

(c)

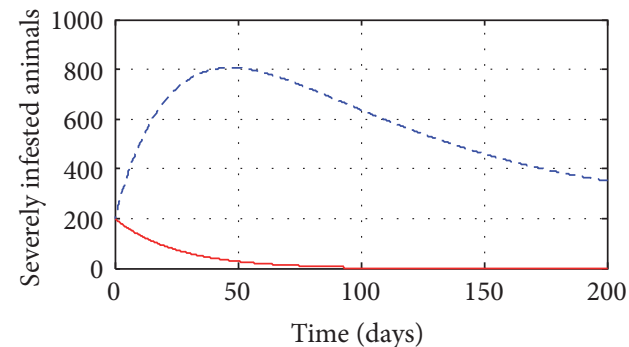

- With opt control

- - - Without control

(b)
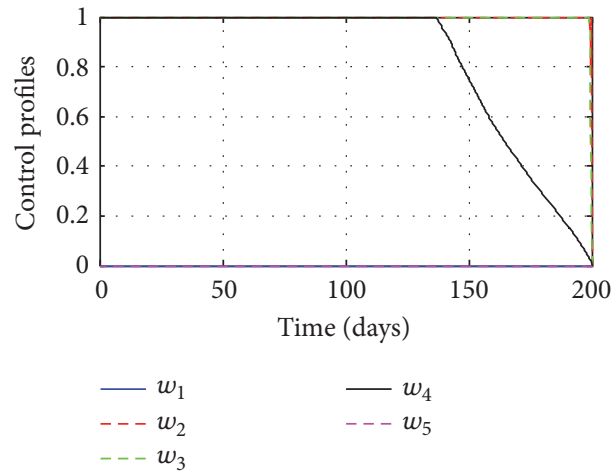

(d)

FIGURE 8: Optimal solutions for model variables $I_{H h}, I_{A h}, F_{E}$ and the control profiles for $w_{1}, w_{2}, w_{3}, w_{4}, w_{5}$ with $\left(w_{2}, w_{3}, w_{4} \neq 0\right)$. 


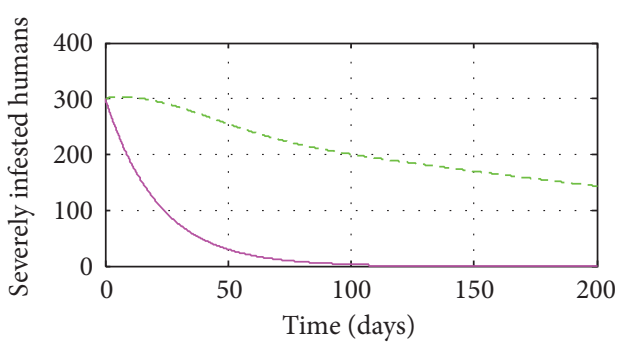

With opt control

- - Without control

(a)

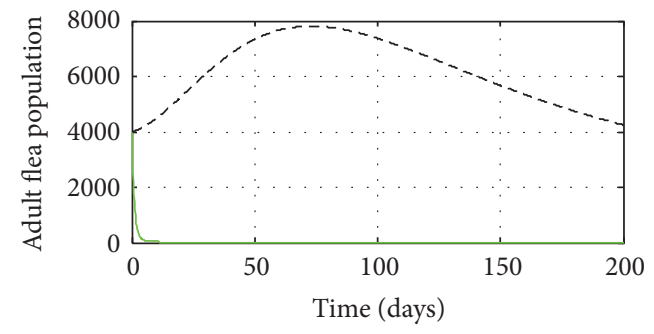

- With opt control

- - Without control

(c)

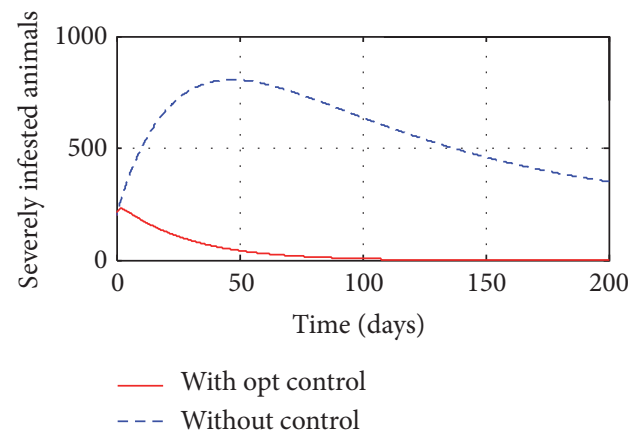

(b)

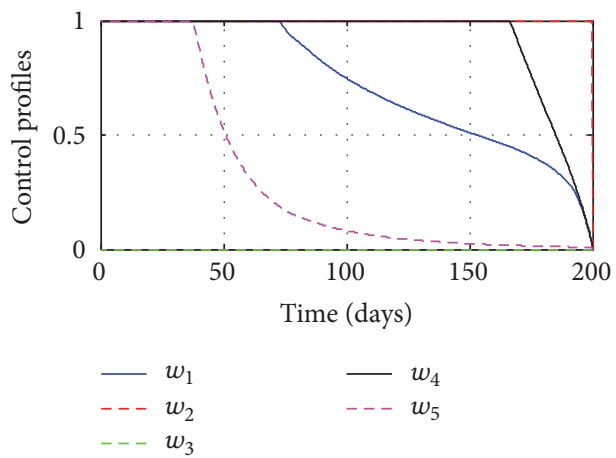

(d)

FIGURE 9: Optimal solutions for model variables $I_{H h}, I_{A h}, F_{E}$ and the control profiles for $w_{1}, w_{2}, w_{3}, w_{4}, w_{5}$ with $\left(w_{1}, w_{2}, w_{4}, w_{5} \neq 0\right)$.

$J(w)$. In Figure 5(d), the control measure $w_{4}$ is at upper bound at the beginning and after 140 days it rapidly drops to the lower bound at the final time. The control measure $w_{5}$ is at the upper bound at the beginning and after 190 days it rapidly drops to the lower bound at the final time. The control measure $w_{3}$ starts at the upper bound at the beginning and remains there until it drops to the lower bound. The control measures $w_{1}$ and $w_{2}$ start at the lower bound at the beginning and remains there till the final time.

4.2.5. Strategy 4: The Control Measures (i.e., $w_{1}, w_{2} \neq 0$ and $w_{3}, w_{4}, w_{5}=0$ ). Under strategy 4 , the control measures $\left(w_{1}, w_{2}\right)$ are used to optimize the objective functional $J(w)$. In Figure 6(d), the control measure $w_{1}$ is at upper bound at the beginning and after 80 days it gradually drops to the lower bound at the final time. The control measure $w_{2}$ starts at the upper bound at the beginning and remains there until it drops to the lower bound. The control measures, $w_{3}, w_{4}$, and $w_{5}$, start at the lower bound at the beginning and remain there till the final time.

4.2.6. Strategy 5: The Control Strategies (i.e., $w_{1}, w_{2}, w_{3} \neq 0$ and $w_{4}, w_{5}=0$ ). Under strategy 5 , the control measures $\left(w_{1}, w_{2}, w_{3}\right)$ are used to optimize the objective functional $J(w)$. In Figure $7(\mathrm{~d})$, the control measure $w_{3}$ is at upper bound at the beginning and after 50 days it gradually drops to the lower bound at the final time. The control measure $w_{1}$ is at the upper bound at the beginning and after 60 days it gradually drops to the lower bound at the final time. The control measure $w_{2}$ starts at the upper bound at the beginning and remains there until it drops to the lower bound at the final time. The control measures $w_{4}$ and $w_{5}$ start at the lower bound at the beginning and remains there till the final time.

4.2.7. Strategy 6: The Control Measures (i.e., $w_{2}, w_{3}, w_{4} \neq 0$ and $w_{1}, w_{5}=0$ ). Under strategy 6 , the control measures $\left(w_{2}, w_{3}, w_{4}\right)$ are used to optimize the objective functional $J(w)$. In Figure 8(d), the control measure $w_{4}$ is at upper bound at the beginning and after 140 days it rapidly drops to the lower bound at the final time. The control measures $w_{2}$ and $w_{3}$ start at the upper bound at the beginning and remain there until they drop to the lower bound. The control measures $w_{1}$ and $w_{5}$ start at the lower bound at the beginning and remain there till the final time.

4.2.8. Strategy 7: The Control Measures (i.e., $w_{1}, w_{2}, w_{4}, w_{5} \neq$ 0 and $w_{3}=0$ ). Under strategy 7 , the control measures $\left(w_{1}, w_{2}, w_{4}, w_{5}\right)$ are used to optimize the objective functional $J(w)$. In Figure 9(d), the control measure $w_{5}$ is at the upper bound at the beginning and after 40 days it gradually drops to the lower bound at the final time. The control measure $w_{1}$ is at the upper bound at the beginning and after 75 days it gradually drops to the lower bound at the final time. The control measure $w_{4}$ is at the upper bound at the beginning and after 170 days it rapidly drops to the lower bound at the final time. The control measure $w_{2}$ starts at the upper bound at the beginning and remains there until it drops to the lower bound at the final time and the control measure $w_{3}$ starts at the lower bound at the beginning and remains there till the final time. 

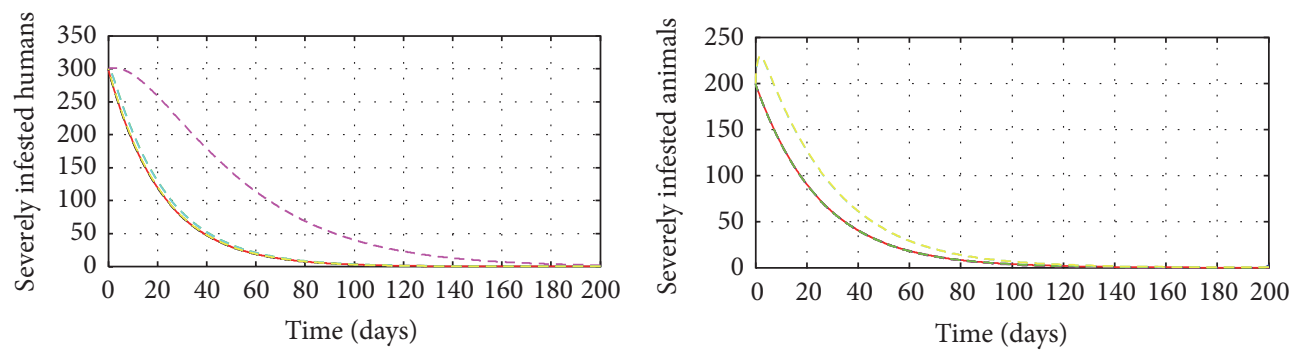

$\begin{array}{ll}\text { - Strategy } 1 & --- \text { Strategy } 5 \\ --- \text { Strategy } 2 & --- \text { Strategy } 6 \\ --- \text { Strategy } 3 & --- \text { Strategy } 7 \\ --- \text { Strategy } 4 & \end{array}$

(a) The comparison of the control strategies on severely infested humans

$\begin{array}{ll}\text { - Strategy } 1 & --- \text { Strategy } 5 \\ \text { - - - Strategy } 2 & --- \text { Strategy } 6 \\ \text { - - - Strategy } 3 & --- \text { Strategy } 7 \\ --- \text { Strategy } 4 & \end{array}$

(b) The comparison of the control strategies on severely infested animals

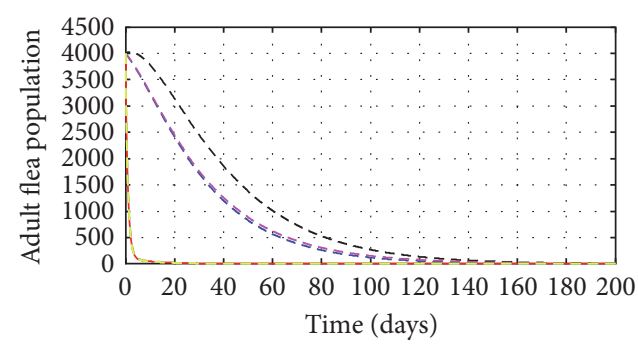

$\begin{array}{ll}\text { - Strategy } 1 & --- \text { Strategy } 5 \\ \text { - - - Strategy } 2 & --- \text { Strategy } 6 \\ \text { - - - Strategy } 3 & --- \text { Strategy } 7 \\ \text { - - - Strategy } 4 & \end{array}$

(c) The comparison of the control strategies on the adult flea population

Figure 10

Generally, it is observed in Figures 3(a)-3(c), 4(a)-4(c), 5(a)-5(c), 6(a)-6(c), 7(a)-7(c), 8(a)-8(c), and 9(a)-9(c) that the controlled trajectories represented by the solid lines decrease compared to uncontrolled trajectories represented by the dotted lines. This implies that the control strategies have the positive impacts which lead to a reduction in the number of infested humans and animals and also reduces the sand flea population in the soil environment.

4.2.9. The Comparison of the Control Strategies. To compare the performance of the control strategies under consideration, we plot the results on same graphs indicating the effects of the control strategies as indicated in Figures 10(a)-10(c).

From Figures 10(a)-10(c), it is observed that the control strategy involving the combination of all the five control measures $w_{1}, w_{2}, w_{3}, w_{4}, w_{5}$ has the significant impact on the reduction of disease transmission because it lowers the severely infested humans and animals and the sand flea populations to minimal levels compared to other control strategies. The red solid line as depicted in Figures 10(a), 10(b), and 10(c) represents the effect caused by the control strategy 1: with the combination of all five control measures. This is the best control strategy because it dominates both graphs as it decreases rapidly compared to other trajectories. Moreover we have observed that those control strategies, whose combinations involve the control measure based on insecticides applications to the premises $\left(w_{1}\right)$, yield better results. These are control strategies 5,6 , and 7 . But control strategy 5 denoted by the control measures $\left(w_{1}, w_{2}, w_{3}\right)$ performs better in the same way as the control strategy 1 denoted by the control measures $\left(w_{1}, w_{2}, w_{3}, w_{4}, w_{5}\right)$.

\section{Conclusion}

In this paper, the optimal control techniques have been applied on Tungiasis dynamical model with control strategies. We defined the control set including controlling the transmission of infestation from flea infested soil environment to human population, from the flea infested soil environment to animal population, and from flea infested animal to human population, controlling flea infested soil environment, and controlling the flea infested animal population. We proved the existence of optimal control problem and determined the necessary conditions for optimality using Pontryagin's maximum principle which converts constrained optimization problem into unconstrained Hamiltonian function whereby optimality and adjoint equations are obtained. We, lastly, performed numerical simulations of the resulting control problem to investigate the effects of the control strategies under consideration and compare their 
performances. The numerical results showed that the control strategy that comprises all five control measures and that with control measures $\left(w_{1}, w_{2}, w_{3}\right)$ have the significant impact on reduced Tungiasis transmission and those control strategies involving insecticides control to the premises $\left(w_{1}\right)$ yielded better results, which implies that the insecticides application control is more effective than other individual control measures. In poor rural communities where resources are always scarce, we suggest that the combination option involving the controls of focal insecticides spraying, insecticidal dusting on animal furs, and environmental hygiene should be adopted, having observed from the comparison of all seven control strategies in Figures 10(a), 10(b), and 10(c) that there is no significant difference between this strategy $\left(w_{1}, w_{2}, w_{3} \neq 0\right)$ and the strategy that involves the combination of the five control measures $\left(w_{1}, w_{2}, w_{3}, w_{4}, w_{5} \neq 0\right)$. Among others, poor housing conditions and the presence of domestic and sylvatic animals on the home compound are risk factors. Therefore, controlling of infested soils and animal reservoirs with insecticides control, ant-flea compounds or animal furs, environmental hygiene, and cementing the floors of houses may serve as a possible approach to control the epidemic and, thus, to fight against Tungiasis infestation in endemic settings multidimensional control process should be employed in order to achieve the maximum benefits.

\section{Notations}

The State Variables of the Model with Control Measures (Source: Kahuru et al. [19])

\begin{tabular}{|c|c|}
\hline$S_{H}(t):$ & $\begin{array}{l}\text { Number of humans in a susceptible class } \\
\text { at time } t\end{array}$ \\
\hline$S_{A}(t):$ & $\begin{array}{l}\text { Number of animals in a susceptible class at } \\
\text { time } t\end{array}$ \\
\hline$I_{H l}(t):$ & $\begin{array}{l}\text { Number of humans in mildly infested } \\
\text { class at time } t\end{array}$ \\
\hline$I_{A l}(t):$ & $\begin{array}{l}\text { Number of animals in mildly infested class } \\
\text { at time } t\end{array}$ \\
\hline$I_{H h}(t):$ & $\begin{array}{l}\text { Number of humans in severely infested } \\
\text { class at time } t\end{array}$ \\
\hline$I_{A h}(t):$ & $\begin{array}{l}\text { Number of animals in severely infested } \\
\text { class at time } t\end{array}$ \\
\hline$T_{H}(t):$ & $\begin{array}{l}\text { Number of humans who are receiving } \\
\text { treatments at time } t\end{array}$ \\
\hline$F_{E}(t):$ & $\begin{array}{l}\text { The density of fleas population in the } \\
\text { environment at time } t\end{array}$ \\
\hline$L_{E}(t):$ & $\begin{array}{l}\text { The density of larvae population in the } \\
\text { environment at time } t\end{array}$ \\
\hline$N_{H}(t), N_{A}(t):$ & $\begin{array}{l}\text { Total human and animal reservoirs } \\
\text { populations at time } t\end{array}$ \\
\hline
\end{tabular}

The Parameters of the Model with Control Measures (Source: Kahuru et al. [19])

$K$ : Maximal larval carrying capacity

$k$ : Half saturation constant

$\gamma_{L}$ : Maturation (transition) rate from larvae to adult jigger fleas

$\begin{array}{ll}\sigma_{H}, \sigma_{A}: & \begin{array}{l}\text { Disease induced death rates for humans } \\ \text { and animal reservoirs, respectively }\end{array} \\ \mu_{H}, \mu_{A}, \mu_{F}, \mu_{L}: \begin{array}{l}\text { Natural mortality rates for humans, } \\ \text { animals, fleas, and larvae, respectively }\end{array} \\ r_{F}: & \begin{array}{l}\text { The rate of removal of jigger fleas that } \\ \text { leaves the soil to attack the hosts }\end{array} \\ \beta_{E H}: & \begin{array}{l}\text { Effective contact rate between } \\ \text { environment and humans }\end{array} \\ \beta_{E A}: & \begin{array}{l}\text { Effective contact rate between } \\ \text { environment and animal reservoirs }\end{array} \\ \rho_{A H}: & \begin{array}{l}\text { Effective contact rate between infested } \\ \text { animals and susceptible humans }\end{array} \\ \rho_{A}: & \begin{array}{l}\text { Effective contact rate between infested } \\ \text { animals and susceptible animals }\end{array} \\ b_{H}, b_{A}: & \begin{array}{l}\text { Recruitment birth rates for humans and } \\ \text { animal reservoirs, respectively }\end{array} \\ \delta_{e}: & \begin{array}{l}\text { The rate of flea eggs deposit on the ground } \\ \text { Shedding rates for adult fleas into the } \\ \varepsilon_{A}:\end{array} \\ \alpha_{E H}, \alpha_{E A}: & \begin{array}{l}\text { Thvironment } \\ \text { and animals, respectively }\end{array} \\ \omega: & \begin{array}{l}\text { Progression rate of treated humans to } \\ \text { susceptible class } \\ \text { Progression rates from infested human } \\ \text { classes to the treatment class. }\end{array} \\ p_{1}, p_{2}: & \end{array}$

\section{Conflicts of Interest}

The authors declare that there are no conflicts of interest.

\section{References}

[1] D. Pilger, S. Schwalfenberg, J. Heukelbach et al., "Controlling tungiasis in an impoverished community: an intervention study," PLoS Neglected Tropical Diseases, vol. 2, no. 10, article e324, 2008.

[2] J. Heukelbach, T. Wilcke, G. Harms, and H. Feldmeier, "Seasonal variation of tungiasis in an endemic community," American Journal of Tropical Medicine and Hygiene, vol. 72, no. 2, pp. 145-149, 2005.

[3] J. Heukelbach, A. M. L. Costa, T. Wilcke, N. Mencke, and H. Feldmeier, "The animal reservoir of Tunga penetrans in severely affected communities of north-east Brazil," Medical and Veterinary Entomology, vol. 18, no. 4, pp. 329-335, 2004.

[4] G. Collins, T. McLeod, N. I. Konfor, C. B. Lamnyam, L. Ngarka, and N. L. Njamnshi, "Tungiasis: a neglected health problem in rural cameroon," International Journal of Collaborative Research on Internal Medicine and Public Health, vol. 1, no. 1, pp. 2-10, 2009.

[5] P. Nordin, M. Thielecke, N. Ngomi, G. M. Mudanga, I. Krantz, and H. Feldmeier, "Treatment of tungiasis with a two-component dimeticone: a comparison between moistening the whole foot and directly targeting the embedded sand fleas," Tropical Medicine and Health, vol. 45, no. 1, 2017.

[6] M. Ozair, A. A. Lashari, I. H. Jung, and K. O. Okosun, "Stability analysis and optimal control of a vector-borne disease with nonlinear incidence," Discrete Dynamics in Nature and Society, vol. 2012, Article ID 595487, 21 pages, 2012.

[7] B. Seidu and O. D. Makinde, "Optimal control of HIV/AIDS in the workplace in the presence of careless individuals," 
Computational and Mathematical Methods in Medicine, Article ID 831506, 19 pages, 2014.

[8] S. M. Lenhart and J. T. Workman, Optimal Control Applied to Biological Models, CRC Press, 2007.

[9] H. S. Rodrigues, M. T. T. Monteiro, and D. F. Torres, "Optimal control and numerical software: an overview," https://arxiv.org/ abs/1401.7279.

[10] H. R. Joshi, S. Lenhart, M. Y. Li, and L. Wang, "Optimal control methods applied to disease models," in Mathematical studies on human disease dynamics, vol. 410 of Contemp. Math., pp. 187207, Amer. Math. Soc., Providence, RI, 2006.

[11] P. D. Roberts and V. M. Becerra, "Optimal control of a class of discrete-continuous non-linear systems-decomposition and hierarchical structure," Automatica. A Journal of IFAC, the International Federation of Automatic Control, vol. 37, no. 11, pp. 1757-1769, 2001.

[12] H. Schättler and U. Ledzewicz, "The Pontryagin Maximum Principle: From Necessary Conditions to the Construction of an Optimal Solution," in Geometric Optimal Control, vol. 38 of Interdisciplinary Applied Mathematics, pp. 83-194, Springer New York, New York, NY, 2012.

[13] P. Ruhnau and C. Schnörr, "Optical stokes flow estimation: an imaging-based control approach," PAMM, vol. 6, no. 1, pp. 863864, 2006.

[14] UNICEF, “State of The World's Children 2015 Country Statistical Information," 2015.

[15] H. D. Gaff, D. M. Hartley, and N. P. Leahy, "An epidemiological model of rift valley fever," Electronic Journal of Differential Equations, vol. 115, pp. 1-12, 2007.

[16] M. Eisele, J. Heukelbach, E. Van Marck et al., "Investigations on the biology, epidemiology, pathology and control of Tunga penetrans in Brazil: I. Natural history of tungiasis in man," Parasitology Research, vol. 90, no. 2, pp. 87-99, 2003.

[17] M. Allerson, J. Deen, S. E. Detmer et al., “The impact of maternally derived immunity on influenza A virus transmission in neonatal pig populations," Vaccine, vol. 31, no. 3, pp. 500-505, 2013.

[18] Tanzania population 2016, “Tanzania Population 2017," http:// worldpopulationreview.com/countries/tanzania-population/.

[19] J. Kahuru, L. Luboobi, and Y. Nkansah-Gyekye, "Modelling the dynamics of Tungiasis transmission in zoonotic areas," Journal of Mathematical and Computational Science, vol. 7, no. 2, pp. 375-399, 2017.

[20] E. Bonyah, I. Dontwi, and F. Nyabadza, "Optimal control applied to the spread of buruli uclcer disease," American Journal of Computational and Applied Mathematics, vol. 4, no. 3, pp. 6167, 2014.

[21] A. O. Isere, J. E. Osemwenkhae, and D. Okuonghae, "Optimal control model for the outbreak of cholera in Nigeria," African Journal of Mathematics and Computer Science Research, vol. 7, no. 2, pp. 24-30, 2014.

[22] G. Devipriya and K. Kalaivani, "Optimal control of multiple transmission of water-borne diseases," International Journal of Mathematics and Mathematical Sciences, vol. 2012, Article ID 421419, 2012.

[23] R. L. M. Neilan, Optimal Control Applied to Population and Disease Models [Ph.D. Dissertation], University of Tennessee, Knoxville, Tennessee, Tenn, USA, 2009.

[24] D. Kirschner, S. Lenhart, and S. Serbin, "Optimal control of the chemotherapy of HIV," Journal of Mathematical Biology, vol. 35, no. 7, pp. 775-792, 1997.
[25] W. H. Fleming and R. W. Rishel, Deterministic and Stochastic Optimal Control, vol. 1, Springer, New York, NY, USA, 1975.

[26] D. L. Lukes, Differential Equations: Classical to Controlled, Mathematics in Science and Engineering, Academic Press, New York, NY, USA, 1982.

[27] S. F. Sadiq, M. A. Khan, S. Islam, G. Zaman, H. Jung, and S. A. Khan, "Optimal control of an epidemic model of leptospirosis with nonlinear saturated incidences," Annual Research of Review in Biology, vol. 4, no. 3, pp. 560-576, 2014.

[28] H. Namawejje, L. S. Luboobi, D. Kuznetsov, and E. Wobudeya, "Modeling optimal control of rotavirus disease with different control strategies," Journal of Mathematical and Computational Science, vol. 4, no. 5, p. 892, 2014.

[29] G. Birkhoff and G. Rota, Ordinary Differential Equations, John Wiley \& Sons, New York, NY, USA, 4th edition, 1989.

[30] K. Blayneh, Y. Cao, and H.-D. Kwon, "Optimal control of vectorborne diseases: treatment and prevention," Discrete and Continuous Dynamical Systems B, vol. 11, no. 3, pp. 587-611, 2009. 


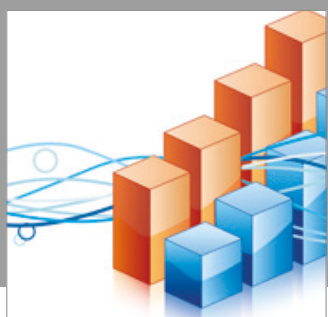

Advances in

Operations Research

vatersals

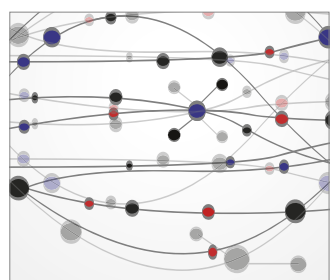

\section{The Scientific} World Journal
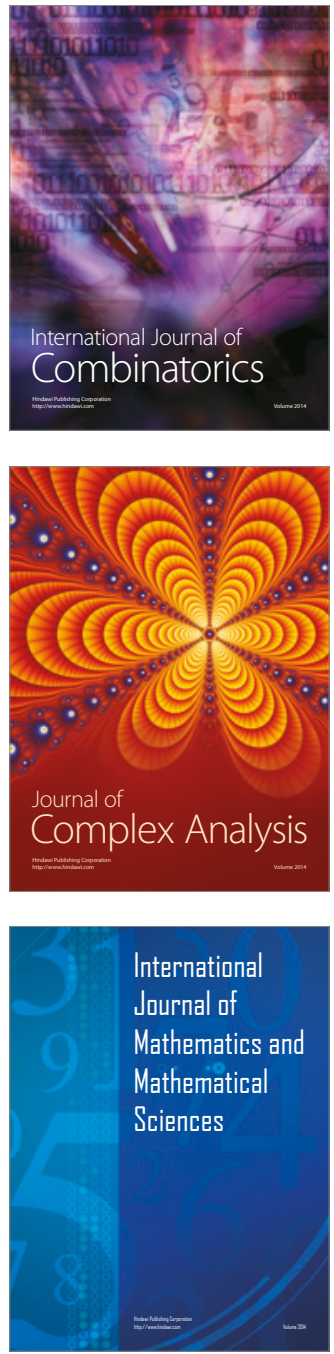
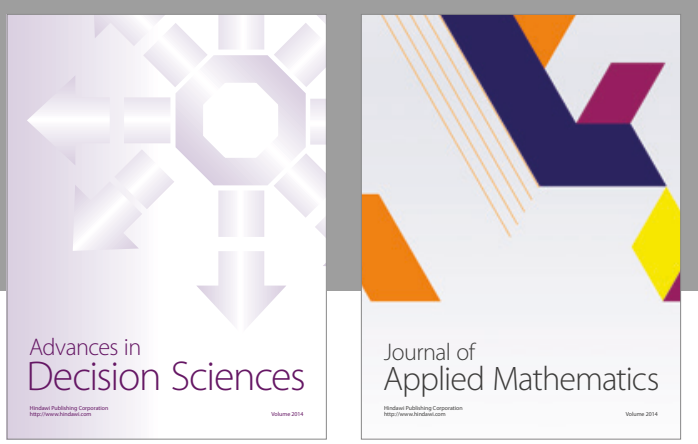

Algebra

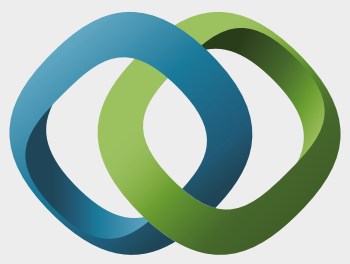

\section{Hindawi}

Submit your manuscripts at

https://www.hindawi.com
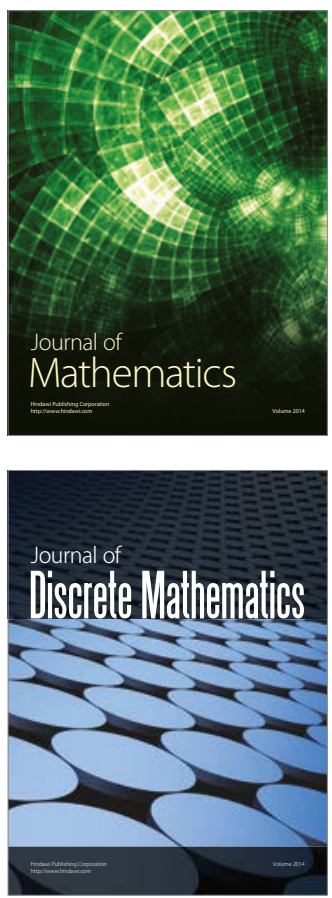

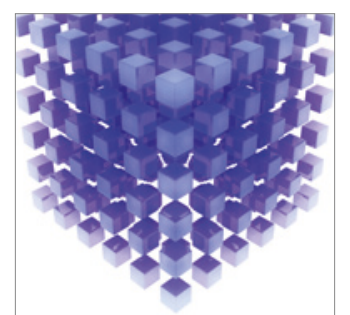

Mathematical Problems in Engineering
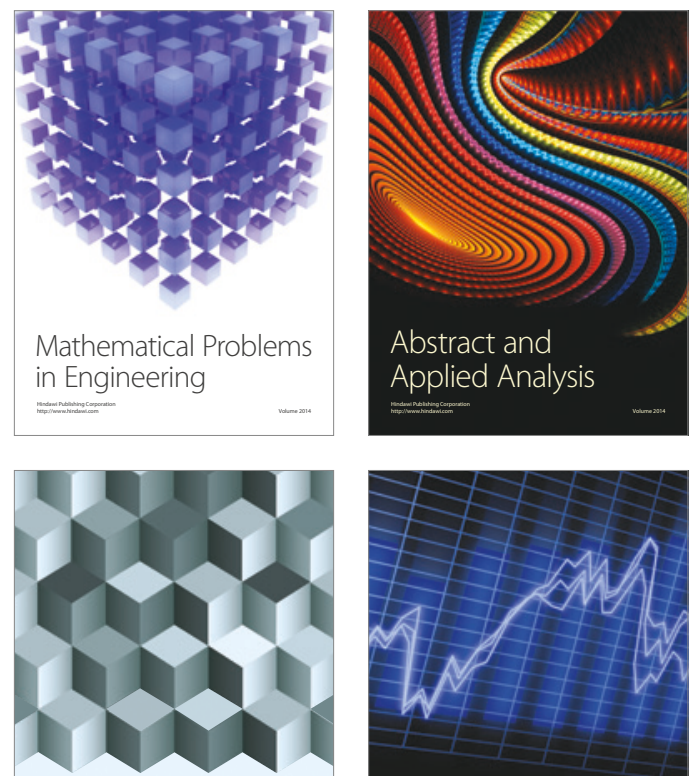

Journal of

Function Spaces

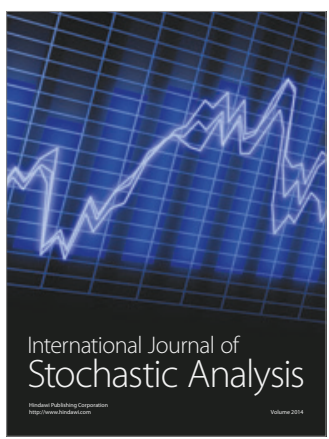

Probability and Statistics
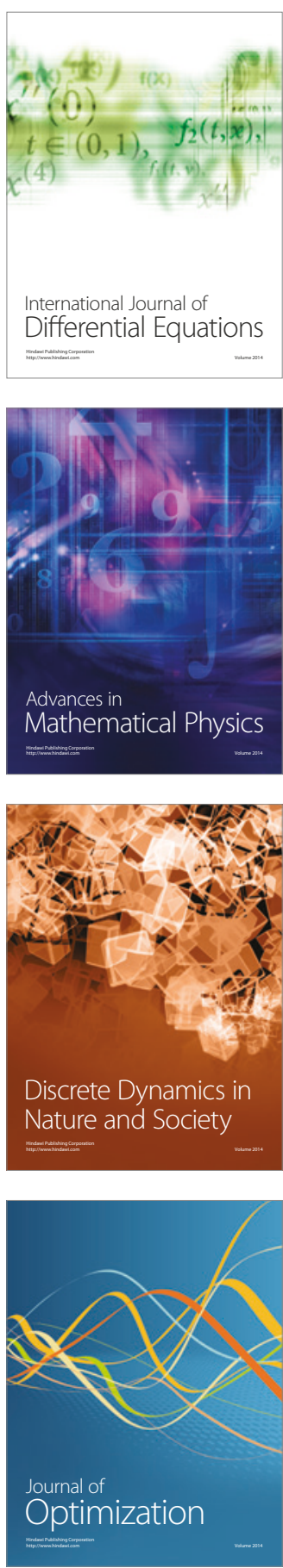